INSTITUT NATIONAL DE LA STATISTIQUE ET DES ETUDES ECONOMIQUES

Série des Documents de Travail du CREST

(Centre de Recherche en Economie et Statistique)

$n^{\circ}$ 2006-28

Pricing and Inference with

Mixtures of Conditionally

Normal Processes

H. BERTHOLON

A. MONFORT 2

F. PEGORARO

Les documents de travail ne reflètent pas la position de l'INSEE et n'engagent que leurs auteurs.

Working papers do not reflect the position of INSEE but only the views of the authors.

\footnotetext{
1 CNAM and IRIA. bertholo@cnam.fr and henri.bertholon@inrialpes.fr

2 CNAM and CREST, Laboratoire de Finance-Assurance. monfort@cnam.fr and monfort@ensae.fr

3 Banque de France, Economics and Finance Research Center [DGEI-DIR-RECFIN] and CREST, Laboratoire de Finance-Assurance. Fulvio.pegoraro@banque-france.fr and pegoraro@ensae.fr
} 


\title{
Pricing and Inference with Mixtures of Conditionally Normal Processes
}

\author{
H. Bertholon ${ }^{(1)}$, A. Monfort ${ }^{(2)}$ and F. Pegoraro ${ }^{(3)}$
}

This version : July, 2006

\author{
Abstract \\ Pricing and Inference with Mixtures \\ of Conditionally Normal Processes
}

\begin{abstract}
We consider the problems of derivative pricing and inference when the stochastic discount factor has an exponentialaffine form and the geometric return of the underlying asset has a dynamics characterized by a mixture of conditionally Normal processes. We consider both the static case in which the underlying process is a white noise distributed as a mixture of Gaussian distributions (including extreme risks and jump diffusions) and the dynamic case in which the underlying process is conditionally distributed as a mixture of Gaussian laws. Semi-parametric, non parametric and Switching Regime situations are also considered. In all cases, the risk-neutral processes and explicit pricing formulas are obtained.
\end{abstract}

Keywords : Derivative Pricing, Stochastic Discount Factor, Implied Volatility, Mixture of Normal Distributions, Mixture of Conditionally Normal Processes, Nonparametric Kernel Estimation, Mixed-Normal GARCH Processes, Switching Regime Models.

\section{Résumé}

Valorisation et Inférence à partir de Mélanges de Processus Conditionnellement Gaussiens

On considère le problème de la valorisation et de l'inférence de produits dérivés quand le facteur d'escompte stochastique (SDF) a une forme exponentielle-affine et le rendement géométrique du titre sous-jacent a une dynamique caractérisée par un mélange de processus conditionnellement Gaussiens. On propose des modèles statiques dans lesquels le processus sous-jacent est un bruit blanc avec une distribution mélange de lois Gaussiennes, et des modèles dynamiques dans lesquels le processus sous-jacent a une distribution conditionnelle du type mélange de lois Gaussiens. On étudie aussi des modèles semi-paramétriques, non paramétriques et a changement de régimes. Pour tous ces modèles on détermine les processus risque-neutre et les formules de valorisations sous une forme explicite.

Mots Clés : Valorisation de Dérivés, Facteur d'Escompte Stochastique, Volatilité Implicite, Mélange de Lois Gaussiennes, Mélange de Processus Conditionnellement Gaussiens, Estimation non paramétrique à noyau, Processus GARCH Mélange de Lois Gaussiennes.

JEL number : C1, C5, G1

${ }^{1}$ CNAM [E-mail: bertholo@cnam.fr] and INRIA [E-mail: henri.bertholon@inrialpes.fr].

${ }^{2}$ CNAM [E-mail: monfort@cnam.fr] and CREST, Laboratoire de Finance-Assurance [E-mail: monfort@ensae.fr].

${ }^{3}$ Banque de France, Economics and Finance Research Center [DGEI-DIR-RECFIN; E-mail: Fulvio.PEGORARO@banque-france.fr] and CREST, Laboratoire de Finance-Assurance [E-mail: pegoraro@ensae.fr]. 


\section{Introduction}

The basic option pricing model, proposed by Black and Scholes (1973), assumes that the logarithmic return of the underlying asset follows a normal white noise. It is well-known that the pricing formula derived from this approach is misspecified; in particular that the implied volatilities are not constant, as a function of the strike and of the maturity and, moreover, depend on time. In the literature, two main routes have been followed in order to solve these problems.

The first type of solutions considers various generalizations of the historical distribution of the underlying stochastic process induced by the observation that stock returns are non-normal (left skewed and leptokurtic), return volatilities vary stochastically over time, and returns and their volatilities are correlated. Among these numerous generalizations are :

i) Stochastic volatilities [Hull and White (1987), Scott (1987), Amin and Ng (1993), Heston (1993), Melino and Turnbull (1990), Renault and Touzi (1996), Jones (2003)];

ii) GARCH-type conditional volatilities [see Engle and Mustafa (1992), Bollerslev and Mikkelsen (1996), Duan (1995, 1996, 1999), Heston and Nandi (2000), Duan, Gauthier, Simonato and Sasseville (2004), Christoffersen, Heston and Jacobs (2004), Christoffersen and Jacobs (2004), Christoffersen, Jacobs and Wang (2005), Christoffersen, Elkamhi and Jacobs (2005)];

iii) Jump components in the stock price and in the stochastic volatility dynamics [Merton (1976), Bates (1996a, 1996b, 2000), Duffie, Pan and Singleton (2000), Pan (2002), Chernov, Gallant, Ghysels and Tauchen (2003), Eraker, Johannes and Polson (2003), Broadie, Chernov and Johannes (2004), Eraker (2004), Duan, Ritchken and Sun (2005a, 2005b)];

iv) The variance gamma (VG) process for the dynamics of the logarithm of the stock price [Madan and Seneta (1990), Madan and Milne (1991), Carr, Madan and Chang (1998)];

v) Time-changed Levy processes [Carr and Wu (2004)];

vi) Switching regimes [Naik (1993), Bollen (1998), Garcia and Renault (1998), Billio and Pelizzon (2000), Chourdakis and Tzavalis (2000), Duan, Popova and Ritchken (2002), Garcia, Luger and Renault (2001, 2003)].

The second type of solutions deals directly with the option pricing formula, the implied volatility surfaces or the risk-neutral probability [see, among the others, Madan and Milne (1994), Rubinstein (1994), Bakshi, Cao and Chen (1997), Dumas, Fleming and Whaley (1998), Ghysels, Patilea, Renault and Torrès (1997), Melick and Thomas (1997), Ait-Sahalia and Lo (1998), Bakshi and Madan (2000), Jondeau and Rockinger (2000), Campbell and Li (2002), Cont and da Fonseca (2002), Duan (2002), Ait-Sahalia and Duarte (2003), Carr and Wu (2003), Huang and Wu (2004)]. In this second approach, the link between the historical and the risk-neutral distributions is in general ignored and, therefore, a precise analysis of how the market prices the different sources of risk affecting option prices is missing.

The general conclusion of the above mentioned literature is that key elements for an option pricing model to replicate the cross-sectional patterns and the dynamics of implied volatilities are the introduction of jump-like features in both returns and volatility, correlation between the jumps in returns and volatility, and pricing the risk for volatility and jumps [see Andersen, Benzoni and Lund (2002), Chernov and Ghysels (2000), Pan (2002)].

The purpose of this paper is to propose the Mixtures of Discrete Time Conditionally Normal processes, combined with the stochastic discount factor (SDF) modeling principle, as a global 
discrete time option pricing methodology providing several model specifications able to solve the problems in the Black-Scholes model, namely the lack of normality and the dynamics. Indeed, we explore various specifications of the historical stochastic processes, while providing at the same time explicit risk-neutral distributions of the processes and option pricing formulas. Typically, the risk-neutral distributions of the processes will be found to belong to the same class as the historical distribution and the option pricing formulas will be combinations of Black-Scholes formulas.

The link between the historical and risk-neutral setting is provided by a parametric SDF under an exponential-affine form which has proved useful in many circumstances [see Gerber and Shiu (1994), Bakshi, Kapadia and Madan (2003), Garcia and Renault (1998), Garcia, Luger and Renault (2001, 2003), Garcia, Ghysels and Renault (2004), Gourieroux and Monfort (2006), Gourieroux, Monfort and Polimenis $(2002,2006)]$. The parametric specification of the pricing kernel leads to price the different sources of risk that affect option prices, providing, in this way, the possibility for a more precise knowledge of the risk premia in options [see Pan (2002)].

As indicated above, the basic tools are the Mixtures of Discrete Time Conditionally Normal processes, that is to say, processes $\left\{y_{t}\right\}$ such that $y_{t}$ is Gaussian conditionally to its past values and the present and past values of a discrete valued unobservable process $z_{t}$. Typically, the dynamics of $z_{t}$ will be (a discrete state space) white noise or Markov chain. In this way, we are able to introduce skewness and excess kurtosis in the historical dynamics of the stock return, to generate implied volatility smiles, volatility skews and implied volatility surfaces coherent with observations. Indeed, a discrete change of state in the latent variable $z_{t}$, affecting simultaneously the conditional (historical and risk-neutral) mean and variance of the return process, produce correlated sources of non-normality suggested by the literature.

From a probabilistic point of view, we consider three main situations. In the static case, the process $\left(y_{t}, z_{t}\right)$ is a white noise, and therefore $y_{t}$ is a mixed-normal white noise. In the mixednormal (MN) GARCH case, $z_{t}$ is an exogenous white noise and, conditionally to its own past, $y_{t}$ is distributed as a mixture of Gaussians laws. In the Switching Regime case, $z_{t}$ is an exogenous discrete process (typically a Markov chain) and conditionally to the past of $y_{t}$ and $z_{t}, y_{t}$ is distributed as a mixture of normal distributions. From a statistical point of view, we consider parametric, semi-parametric and nonparametric cases. In the non parametric and semi-parametric cases the normality is introduced in the kernel used at the estimation stage.

The plan of the paper is as follows. In Section 2, we review the use of exponential-affine stochastic discount factor and of real Laplace transform (or moment generating function). In Section 3, we present the advantages of mixtures of normal distributions, and in particular their ability to span the skewness-kurtosis domain of maximal size. In Section 4, we consider the case where the historical process is a white noise distributed as a Gaussian mixture, we present the pricing formula, we consider the special cases of extreme risks and jump diffusions, and we numerically study its ability to replicate implied volatility smiles, volatility skews and volatility surfaces coherent with observations. In Section 5, we study the nonparametric static case, that is the case where the white noise distribution is unspecified. In Section 6, we consider a parametric dynamic case; more precisely, several kinds of conditionally mixed-normal GARCH processes are proposed. In Section 7 , we deal with the semi-parametric dynamic case, in which the distribution of the conditionally standardized process is left unspecified, while, in Section 8, we study the Switching Regime modelisation; here, we present an easy simulation procedure, using the explicit specification we have about the risk-neutral density, to price path dependent derivatives and European options with time to maturity larger than one. Moreover, we compare the implied volatility surface obtained by the Switching Regimes model with the one obtained by the static Mixed-Normal specification. Section 9 concludes and appendices gather the proofs. 


\section{Pricing With Exponential-Affine Stochastic Discount Factor}

In order to briefly present the Stochastic Discount Factor (SDF) modeling principle [see Gourieroux and Monfort (2006) for a detailed presentation], we consider a frictionless market with a riskfree asset and one risky asset; we denote by $r_{t+1}^{f}$ the riskfree rate between the dates $t$ and $t+1$ (known at time $t$, that is, predetermined) and by $y_{t+1}=\ln \left(S_{t+1} / S_{t}\right)$ the geometric return on the risky asset with price $S_{t}$.

In this context, under the absence of arbitrage opportunities, the price at $t$ of a derivative asset paying $g\left(y_{t+1}, \ldots, y_{t+H}\right)$ at $t+H$, can be written (under the historical probability) in the following way :

$$
\begin{aligned}
C_{t}(g, H) & =E\left[M_{t, t+1} \ldots M_{t+H-1, t+H} g\left(y_{t+1}, \ldots, y_{t+H}\right) \mid I_{t}\right] \\
& =E_{t}\left[M_{t, t+H} g\left(y_{t+1}, \ldots, y_{t+H}\right)\right]
\end{aligned}
$$

where $I_{t}=y_{t}=\left(y_{t}, y_{t-1}, \ldots\right)$ is the information on the current and lagged values of the state variable available at date $t$ for the investor, and where $M_{t, t+1}$ is the Stochastic Discount Factor between $t$ and $t+1$, which is function of $I_{t+1}$.

In particular, we present the problem of asset pricing by means of a stochastic discount factor (SDF) $M_{t, t+1}$ characterized by an exponential-affine form :

$$
M_{t, t+1}=\exp \left[\alpha_{t} y_{t+1}+\beta_{t}\right]
$$

where coefficients $\alpha_{t}$ and $\beta_{t}$ can be path dependent, that is, function of $I_{t}$. Observe that this specification of the pricing kernel characterizes power utility economies with a non-constant relative risk-aversion coefficient $\phi_{t}=-\alpha_{t}$ [the same kind of SDF specification with constant coefficients is considered, among the others, by Bakshi, Kapadia and Madan (2003) and Léon, Mencia and Sentana (2005)].

By writing the pricing formula for the riskfree and risky asset at different dates, we obtain two arbitrage free conditions that induce restrictions on the relationship between the SDF and the historical distribution. More precisely, the constraints are :

$$
\begin{gathered}
\left\{\begin{array}{l}
E_{t}\left[M_{t, t+1} \exp r_{t+1}^{f}\right]=1 \\
E_{t}\left[M_{t, t+1} \exp y_{t+1}\right]=1
\end{array}\right. \\
\Longleftrightarrow\left\{\begin{array}{l}
\exp \left(r_{t+1}^{f}+\beta_{t}\right) E_{t}\left[\exp \alpha_{t} y_{t+1}\right]=1 \\
\exp \left(\beta_{t}\right) E_{t}\left[\exp \left(\alpha_{t}+1\right) y_{t+1}\right]=1
\end{array}\right. \\
\Longleftrightarrow\left\{\begin{array}{l}
\exp \left(r_{t+1}^{f}+\beta_{t}\right) \varphi_{t}\left(\alpha_{t}\right)=1 \\
\exp \left(\beta_{t}\right) \varphi_{t}\left(\alpha_{t}+1\right)=1,
\end{array}\right.
\end{gathered}
$$

where $\varphi_{t}(\alpha)$ is the real conditional Laplace transform (also called moment generating function) of $y_{t+1}$ (given $I_{t}$ ).

The Laplace transform, used to describe the conditional historical distribution of $y_{t+1}$, is defined on a convex set that depends on the tails of the conditional distribution. We assume below that 
this convex set is not reduced to one point located at the origin.

This system in general admits a unique solution $\left(\alpha_{t}, \beta_{t}\right)$ such that:

$$
\left\{\begin{array}{l}
\varphi_{t}\left(\alpha_{t}+1\right)=\exp r_{t+1}^{f} \varphi_{t}\left(\alpha_{t}\right) \\
\exp \beta_{t}=\left[\varphi_{t}\left(\alpha_{t}+1\right)\right]^{-1}
\end{array}\right.
$$

and, consequently, we deduce a unique specification of the SDF (2.2).

The associated unique risk-neutral conditional distribution $Q_{t}$ of $y_{t+1}$, given $I_{t}$, has a p.d.f. with respect to the corresponding historical distribution given by $M_{t, t+1} / E_{t}\left(M_{t, t+1}\right)$ and a Laplace transform given by:

$$
\begin{aligned}
& E^{Q_{t}}\left[\exp u y_{t+1}\right] \\
& =E_{t}\left[\frac{M_{t, t+1}}{E_{t}\left(M_{t, t+1}\right)} \exp u y_{t+1}\right] \\
& =\exp \left(r_{t+1}^{f}+\beta_{t}\right) E_{t}\left[\exp \left(\left(\alpha_{t}+u\right) y_{t+1}\right)\right] \\
& =\frac{\varphi_{t}\left(\alpha_{t}+u\right)}{\varphi_{t}\left(\alpha_{t}\right)}
\end{aligned}
$$

An asset providing the payoff $g\left(y_{t+1}\right)$ at time $t+1$ is priced at time $t$ by :

$$
C_{t}(g, 1)=E_{t}\left[M_{t, t+1} g\left(y_{t+1}\right)\right]=\exp \left(-r_{t+1}^{f}\right) E^{Q t}\left[g\left(y_{t+1}\right)\right] .
$$

With a larger time horizon $H$, the conditional joint risk-neutral distribution $Q_{t}^{H}$ of $\left(y_{t+1}, \ldots, y_{t+H}\right)$ given $I_{t}$ has a p.d.f., with respect to the corresponding historical distribution $P_{t}^{H}$, given by :

$$
\frac{d Q_{t}^{H}}{d P_{t}^{H}}=\frac{M_{t, t+1} \cdot \ldots \cdot M_{t+H-1, t+H}}{E_{t}\left(M_{t, t+1}\right) \cdot \ldots \cdot E_{t+H-1}\left(M_{t+H-1, t+H}\right)},
$$

and the associated pricing formula takes the form :

$$
\begin{aligned}
C_{t}(g, H) & =E_{t}\left[M_{t, t+1} \cdot \ldots \cdot M_{t+H-1, t+H} g\left(y_{t+1}, \ldots, y_{t+H}\right)\right] \\
& =E^{Q_{t}}\left[E_{t}\left(M_{t, t+1}\right) \cdot \ldots \cdot E_{t+H-1}\left(M_{t+H-1, t+H}\right) g\left(y_{t+1}, \ldots, y_{t+H}\right)\right] .
\end{aligned}
$$

If the short term riskfree rates are known at date $t$, we get :

$$
C_{t}(g, H)=\exp \left(-\sum_{h=1}^{H} r_{t+h}\right) E^{Q t}\left[g\left(y_{t+1}, \ldots, y_{t+H}\right)\right]
$$




\section{The advantages of Mixtures of Normal distributions}

\subsection{Flexibility of Mixed-Normal Statistical models}

It is well known from empirical research that, contrary to the Gaussian case, the distributions of stock returns are characterized by a non zero skewness and a large kurtosis [see Mandelbrot (1962, 1963a,b, 1967), Fama (1965)], and in order to capture these features several distributions have been proposed in the literature. Families of distributions that have shown a close data fit are the Stable Paretian distributions [see, for example, Mandelbrot (1997), Mittnick and Rachev (1993a,b), Mittnick, Paolella and Rachev (1997), Adler et al. (1998)], the Finite Mixture of Normal distributions [see, among the others, Kon (1984), Akgiray and Booth (1987), Tucker and Pond (1988)], the Student distributions [see Bollerslev (1987), Baillie and Bollerslev (1989), Palm and Vlaar (1997), Lambert and Laurent $(2000,2001)$ ] and the hyperbolic distributions [see BarndorffNielsen (1994), Eberlein and Keller (1995), Kuechler et al. (1994)]. More recently, Jondeau and Rockinger (2001, 2002, 2003) proposed, respectively, Gram-Charlier, Entropy and Generalized Student- $t$ densities, while, Leon, Mencia and Sentana (2005) proposed the semi-nonparametric distribution (SNP) introduced by Gallant and Nychka (1987).

Our choice of a mixture of Normal distributions as a basic tool for the modelling of stock returns derives from the following important properties :

i) it encompasses the Normal distribution and it can be used to model a continuous distortion of the latter by means of two or several weights [see e.g. contamination models in Section 4.2, or jump diffusion models in Section 4.3].

ii) it can approximate any kind of distributions since the well-known Normal kernel density estimator may be viewed as a particular mixture of Normal distributions [see also Section 5].

iii) it is stable by convolution [which is convenient for example when summing geometric returns on several periods].

iv) it is very easy to simulate.

v) it matches well with theoretical tools such as Laplace Transforms, and therefore it is adapted for option pricing purposes [see Section 4].

Moreover, other important features of the mixtures of Normal distributions are presented below.

\subsection{Spanning the skewness-kurtosis domain of maximal size}

It is well known that the skewness $\tilde{\mu}_{3}$ and kurtosis $\tilde{\mu}_{4}$ of a random variable with any probability distribution span the domain $\mathcal{D}=\left\{\left(\tilde{\mu}_{3}, \tilde{\mu}_{4}\right) \in \mathbb{R} \times \mathbb{R}_{+}^{*}: \tilde{\mu}_{4} \geq \tilde{\mu}_{3}^{2}+1\right\}$. This means that, in the $\left(\tilde{\mu}_{3}, \tilde{\mu}_{4}\right)$-plane, the boundary of the skewness-kurtosis domain of maximal size $\mathcal{D}$ is a parabola in which $\tilde{\mu}_{4}$ is bounded from below by 1 .

The purpose of this section is to show that any possible pair of skewness-kurtosis in the maximal set $\mathcal{D}$ can be reached by a mixture of only two Normal distributions ${ }^{4}$. Moreover, we provide (quasiexplicit) parameter values of the mixture able to replicate any given mean $\mu$, variance $\sigma^{2}$, skewness $\tilde{\mu}_{3}$ and kurtosis $\tilde{\mu}_{4}$.

\footnotetext{
${ }^{4}$ Jondeau and Rockinger (2001, 2003), and Leon, Mencia and Sentana (2005) also studied the set of skewnesskurtosis pairs spanned by their densities. In all these cases, only a (bounded or unbounded) subset of $\mathcal{D}$ was reached.
} 
Proposition 1 : The family of mixtures of two Normal distributions spans $\mathcal{D}$. More precisely, we have the following two cases.

Case 1 : The mixture of two normal distributions :

$$
p \mathcal{N}\left(\mu+\sigma \sqrt{\frac{1-p}{a p}}, \frac{\sigma^{2}(a-1)}{a}\right)+(1-p) \mathcal{N}\left(\mu-\sigma \sqrt{\frac{p}{a(1-p)}}, \frac{\sigma^{2}(a-1)}{a}\right)
$$

where :

- $a$ is the (unique) root $\geq 1$ of the polynomial $p(x)=\tilde{\mu}_{3}^{2} x^{3}+\left(3-\tilde{\mu}_{4}\right) x^{2}-2$

- $p=\frac{1}{2}-\frac{\tilde{\mu}_{3}^{o}}{2 \sqrt{\left(\tilde{\mu}_{3}^{o}\right)^{2}+4}}$, with $\tilde{\mu}_{3}^{o}=a^{\frac{3}{2}} \tilde{\mu}_{3}$.

span the set $\left(\mu, \sigma^{2}, \tilde{\mu}_{3}, \tilde{\mu}_{4}\right)$ with $\tilde{\mu}_{3} \neq 0$, or $\tilde{\mu}_{3}=0$ and $\tilde{\mu}_{4}<3$.

Case 2 : The mixture of two normal distributions :

$$
p \mathcal{N}\left(\mu, \frac{\sigma^{2}}{2 p}\right)+(1-p) \mathcal{N}\left(\mu, \frac{\sigma^{2}}{2(1-p)}\right)
$$

with $p=\frac{1}{2} \pm \frac{1}{2} \sqrt{1-\frac{3}{\tilde{\mu}_{4}}}$, spans the set $\left(\mu, \sigma^{2}, \tilde{\mu}_{3}, \tilde{\mu}_{4}\right)$ with $\tilde{\mu}_{3}=0$ and $\tilde{\mu}_{4} \geq 3$.

[Proof : see Appendix 1.]

\subsection{Matching financial stylized facts}

In a financial context, two important elements are in favour of the mixed-normal statistical model.

The first element arises from an empirical observation [see, for instance, Campbell, Lo and MacKinlay (1997)] that the Paretian family, for instance, is not able to reproduce. Empirical analysis show that asymmetries and fat-tails are much weaker for low frequency observations (long horizon returns) than for high frequency ones (short horizon returns), that is, the marginal distribution of $y_{t, t+k}=\sum_{i=1}^{k} y_{t+i}, k \in \mathbb{N}$ and $k \geq 1$, where $y_{t}$ is the geometric return of a given risky asset between $t-1$ and $t$, shows decreasing negative skewness and leptokurtosis as $k$ increases. Therefore, if we want to perform this kind of behaviour we need to use distributions characterized by finite moments for which the Central Limit Theorem applies and drives longer-horizon returns towards normality. In particular, empirical researches [see Tucker (1992)] have shown that the general stable Paretian model is dominated by the jump-diffusion model [see Merton (1976) and paragraph 4.3] and by the finite mixture of Normal distributions model; in the latter case, it seems that the higher goodness of fit is obtained by a mixture of two Normal distributions.

The second element is linked to an interesting feature of the Mixed Normal distribution, not shared by other distributions (like, for instance, the hyperbolic or the Student distribution), that is, the economic interpretation we can give to it and that can be of interest not only for researchers, but also for practitioners such as risk managers. For instance, a mixture of two or more Normal distributions is used, in the asset pricing literature, to describe different regimes characterizing the fundamentals of the economy (consumption or dividend growth rate), or to represent market periods with different levels of volatility. Moreover, this modelling offers the possibility to better explain a number of phenomena like the return predictability or the relation between risk and 
return [see, among the others, Abel (1994, 1999), Bonomo and Garcia (1994, 1996) Cecchetti, Lam and Mark (1990), Veronesi (1999, 2000, 2004), Whitelaw (2000), Calvet and Fisher (2004), Garcia, Meddahi, Tedongap (2006)].

The Mixed-Normal distribution will be the basic building block of this paper, both for static models and dynamic models.

\section{The Static Parametric Model}

In this Section, we study the pricing problem through a static parametric model. More precisely, in Section 4.1, we consider the case where the geometric returns $y_{t}$ of the risky assets are i.i.d. and distributed as a finite mixture of Normal distributions. So the process $y_{t}$ can be viewed as Gaussian conditional on the discrete valued white noise giving at each date $t$ the index of the relevant Gaussian component. The riskless rate is fixed and denoted by $r$. In Section 4.2, we use the mixed normal distribution for modeling extreme risks whereas, in Section 4.3, we present the jump-diffusion case (infinite countable mixture of Normal distributions).

\subsection{Pricing with a finite mixture of Normal distributions}

\subsubsection{Historical and risk-neutral distributions}

Since, in this section we are in a static case, we will often drop the index $t$ for sake of notational simplicity. Let us consider a geometric return $y$ whose historical distribution is a mixture of $J$ Normal distributions denoted by $\mathcal{M N}\left(J, p_{j}, \mu_{j}, \sigma_{j}^{2}\right)$, its p.d.f. is given by :

$$
f(y)=\sum_{j=1}^{J} p_{j} n\left(y ; \mu_{j}, \sigma_{j}^{2}\right)
$$

where, for $j=1, \ldots, J$

$$
\begin{gathered}
n\left(y ; \mu_{j}, \sigma_{j}^{2}\right)=\frac{1}{\sigma_{j} \sqrt{2 \pi}} e^{-\frac{1}{2} \frac{\left(y-\mu_{j}\right)^{2}}{\sigma_{j}^{2}}}, \\
0 \leq p_{j} \leq 1, \sum_{j=0}^{J} p_{j}=1 .
\end{gathered}
$$

Its mean, variance, skewness and kurtosis are 


$$
\begin{aligned}
\mu & =\sum_{j=1}^{J} p_{j} \mu_{j} \\
\sigma^{2} & =\sum_{j=1}^{J} p_{j}\left(\sigma_{j}^{2}+\mu_{j}^{2}\right)-\mu^{2} \\
\tilde{\mu}_{3} & =\frac{1}{\sigma^{3}} \sum_{j=1}^{J} p_{j}\left(\mu_{j}-\mu\right)\left[3 \sigma_{j}^{2}+\left(\mu_{j}-\mu\right)^{2}\right] \\
\tilde{\mu}_{4} & =\frac{1}{\sigma^{4}} \sum_{j=1}^{J} p_{j}\left[3 \sigma_{j}^{4}+6\left(\mu_{j}-\mu\right)^{2} \sigma_{j}^{2}+\left(\mu_{j}-\mu\right)^{4}\right] .
\end{aligned}
$$

By applying in this static framework the general approach described in Section 2 we get the following results. In particular, in Proposition 4 we obtain the pricing formula for a Call option with maturity one and strike $K$ : this derivative asset gives the payoff $\left(S_{t+1}-K\right)^{+}=S_{t}\left[\exp y_{t+1}-\kappa\right]^{+}$ where $\kappa=K / S_{t}$. Normalizing $S_{t}$ to one the payoff is $\left(\exp y_{t+1}-\kappa\right)^{+}$.

Proposition 2 : If the historical distribution is a mixture of $J$ Normal distributions $\mathcal{M N}\left(J, p_{j}, \mu_{j}, \sigma_{j}^{2}\right)$ and if the stochastic discount factor is exponential-affine, we have a unique solution $(\alpha, \beta)$ that satisfies system (2.3). The unique value of $\alpha$ is the solution of :

$$
\sum_{j=1}^{J} p_{j} \exp \left(\alpha \mu_{j}+\sigma_{j}^{2} \frac{\alpha^{2}}{2}\right)\left[\exp \left(\mu_{j}+\sigma_{j}^{2} \alpha+\frac{\sigma_{j}^{2}}{2}\right)-\exp r\right]=0 .
$$

[Proof : see Appendix 2.]

Proposition 3 : The risk-neutral distribution $Q$ is unique and is a mixture of Normal distributions with the following p.d.f. :

$$
f^{Q}(y)=\sum_{j=1}^{J} \nu_{j} n\left(y ; \mu_{j}+\alpha \sigma_{j}^{2}, \sigma_{j}^{2}\right)
$$

where, for $j=1, \ldots, J$

$$
\begin{gathered}
\nu_{j}=\frac{p_{j} \exp \left(\alpha \mu_{j}+\sigma_{j}^{2} \frac{\alpha^{2}}{2}\right)}{\sum_{j=1}^{J} p_{j} \exp \left(\alpha \mu_{j}+\sigma_{j}^{2} \frac{\alpha^{2}}{2}\right)} \\
0 \leq \nu_{j} \leq 1, \sum_{j=1}^{J} \nu_{j}=1,
\end{gathered}
$$

and with $\alpha$ the solution of relation (4.2). [Proof : see Appendix 3.]

When $J>1$, the risk-neutral distribution depends on the volatilities $\sigma_{j}$ 's and also on the drifts $\mu_{j}$ 's, with $j \in\{1, \ldots, J\}$, denoting an evolution with respect to the Black-Scholes framework $(J=1)$. 
Proposition 4 : The price of the European Call option with payoff $\left(\exp y_{t+1}-\kappa\right)^{+}$and maturity one is:

$$
\begin{aligned}
C_{t} & =\exp (-r) E^{Q}\left[\exp y_{t+1}-\kappa\right]^{+} \\
& =\sum_{j=1}^{J} \nu_{j} \gamma_{j} C_{B S}\left(\sigma_{j}^{2}, \frac{\kappa}{\gamma_{j}}\right),
\end{aligned}
$$

where $C_{B S}$ is the (one-period) Black-Scholes formula with a volatility equal to $\sigma_{j}^{2}$ and moneyness strike equal to $\kappa / \gamma_{j}$, and

$$
\gamma_{j}=\exp \left(\mu_{j}+\alpha \sigma_{j}^{2}-r+\frac{\sigma_{j}^{2}}{2}\right) .
$$

Moreover, it can be shown that

$$
0 \leq \nu_{j} \gamma_{j} \leq 1, \sum_{j=1}^{J} \nu_{j} \gamma_{j}=1
$$

[Proof : see Appendix 4.]

The propositions presented above consider the case of a one-period geometric return $y_{t+1}$, but similar results can be obtained for a geometric stock return $y_{t, t+H}=y_{t+1}+\ldots+y_{t+H}$ at horizon $H$ larger than one. Indeed, $y_{t, t+H}$ has a distribution which is once more a mixture of normal distributions with historical p.d.f. :

$$
f\left(y_{t, t+H}\right)=\sum_{\substack{h_{j}=0 \\ j=\{1, \ldots, J-1\}}}^{H} \frac{H !}{h_{1} ! \cdot \ldots \cdot h_{J} !} p_{1}^{h_{1}} \cdot \ldots \cdot p_{J}^{h_{J}} n\left(\sum_{j=1}^{J} h_{j} \mu_{j}, \sum_{j=1}^{J} h_{j} \sigma_{j}^{2}\right),
$$

with $\sum_{j=1}^{J} h_{j}=H$.

\subsubsection{Generalizing the Black-Scholes market risk premium}

The pricing formula that we obtain by the finite mixture of Normal distributions assumption is a linear combination of $J$ Black-Scholes formulas. It depends not only on the variances, but also on the means of the Gaussian distributions in the mixture.

Moreover, it is easily seen that, in the Gaussian (Black-Scholes) case (i.e. when $J=1$ ) we have, under the absence of arbitrage restrictions, that $\alpha_{b s}=-\left(\sigma^{2}\right)^{-1}\left[\mu-r+\left(\sigma^{2} / 2\right)\right]$, therefore $\gamma_{j}$ can be written :

$$
\gamma_{j}=\exp \left[\sigma_{j}^{2}\left(\alpha-\alpha_{b s, j}\right)\right]
$$

where $\alpha_{b s, j}=-\left(\sigma_{j}^{2}\right)^{-1}\left[\mu_{j}-r+\left(\sigma_{j}^{2} / 2\right)\right]$ is the value of $\alpha_{b s}$ corresponding to the Gaussian case associated with the $j^{\text {th }}$ component of the mixture, and where $\alpha$ is the risk correction factor associated with the Mixed-Normal framework. Indeed, if we consider the Black-Scholes parameterization of the Gaussian distribution, that is, if we define $\mu_{j}=m_{j}-\sigma_{j}^{2} / 2$, we find that $\alpha_{b s, j}=-\Pi_{b s, j}$, with 
$\Pi_{b s, j}=\left(m_{j}-r\right) / \sigma_{j}^{2}$ the price of risk characterizing the $j^{t h}$ Gaussian market; consequently, we can rewrite $\gamma_{j}$ in the following way :

$$
\gamma_{j}=\exp \left[\sigma_{j}^{2}\left(\Pi_{b s, j}-\Pi_{m n}\right)\right],
$$

where $\Pi_{m n}=-\alpha$ can be interpreted as the price of risk in the Mixed-Normal market.

In particular, the modified BS formula $C_{B S}\left(\sigma_{j}^{2}, \kappa / \gamma_{j}\right)$ defines an option price larger (resp. smaller) than the BS price $C_{B S}\left(\sigma_{j}^{2}, \kappa\right)$ if the coefficient $\gamma_{j}$ modifying the strike is larger (resp. smaller) than one, that is, if $\Pi_{b s, j}$ is larger (resp. smaller) than $\Pi_{m n}$. Thus, if the $j^{\text {th }}$ Gaussian market has a level of risk (priced by $\Pi_{b s, j}$ ) larger (resp. smaller) than the general level (priced by $\Pi_{m n}$ ), then the Mixed-Normal model gives a price larger (resp. smaller) than the price determined by the Black-Scholes model [compare with Ritchey (1990) where a strong assumption of riskneutrality is made].

\subsection{Modeling extreme risks}

Here we are interested in a particular mixture of two normal distributions, one of which having a small weight associated with a strong variance. This example enables us to take into account the unlikely occurrence of an important shock on the volatility.

More precisely, we consider a geometric return $y$ whose distribution is given by the following p.d.f :

$$
f(y)=\lambda n\left(y ; \mu, \frac{\sigma_{1}^{2}}{\lambda}\right)+(1-\lambda) n\left(y ; \mu, \frac{\sigma_{2}^{2}}{1-\lambda}\right),
$$

and we consider the situation in which $\lambda$ tends to zero.

Proposition 5 : The main characteristics of this distribution are:

$$
\begin{gathered}
E(y)=\mu \\
\sigma^{2}=V(y)=\sigma_{1}^{2}+\sigma_{2}^{2} \\
\frac{E(y-\mu)^{3}}{\sigma^{3}}=0 \\
\frac{E(y-\mu)^{4}}{\sigma^{4}}=\frac{3\left[\frac{\sigma_{1}^{4}}{\lambda}+\frac{\sigma_{2}^{4}}{1-\lambda}\right]}{\left[\sigma_{1}^{2}+\sigma_{2}^{2}\right]^{2}} \rightarrow+\infty \\
y \stackrel{D}{\longrightarrow} \mathcal{N}\left(\mu, \sigma_{2}^{2}\right), \text { when } \lambda \rightarrow 0 .
\end{gathered}
$$

[Proof : see Appendix 5.]

These results show that, when $\lambda$ tends to 0 , this distribution is in a sense close to the Gaussian distribution, but with a strong kurtosis; it is a case where the convergence in distribution does not imply the convergence of the moments. 
We now apply the general propositions of Section 4.1.1 to this special case [see Appendix 5 for the proofs].

Proposition 6 : The value of $\alpha$ given by the solution of equation (4.2) converges to $-\frac{1}{2}$ when $\lambda \rightarrow 0$.

Proposition 7 : The risk-neutral distribution $Q$ has the following p.d.f. :

$$
f^{Q}(y)=\nu_{1} n\left(y ; \mu+\alpha \frac{\sigma_{1}^{2}}{\lambda}, \frac{\sigma_{1}^{2}}{\lambda}\right)+\nu_{2} n\left(y ; \mu+\alpha \frac{\sigma_{2}^{2}}{1-\lambda}, \frac{\sigma_{2}^{2}}{1-\lambda}\right)
$$

where

$$
\nu_{1}=\frac{\lambda \exp \left(\frac{\sigma_{1}^{2}}{\lambda} \frac{\alpha^{2}}{2}\right)}{\lambda \exp \left(\frac{\sigma_{1}^{2}}{\lambda} \frac{\alpha^{2}}{2}\right)+(1-\lambda) \exp \left(\frac{\sigma_{2}^{2}}{1-\lambda} \frac{\alpha^{2}}{2}\right)} \quad \text { and } \quad \nu_{2}=1-\nu_{1}
$$

We note that $: \quad \nu_{1} \longrightarrow 1$ when $\quad \lambda \rightarrow 0$.

Proposition 8 : The price of the European Call option can be written :

$$
C_{t}=\nu_{1} \gamma_{1} C_{B S}\left(\frac{\sigma_{1}^{2}}{\lambda}, \frac{\kappa}{\gamma_{1}}\right)+\nu_{2} \gamma_{2} C_{B S}\left(\frac{\sigma_{2}^{2}}{1-\lambda}, \frac{\kappa}{\gamma_{2}}\right)
$$

with

$$
\begin{aligned}
& \gamma_{1}=\exp \left(\mu+\alpha \frac{\sigma_{1}^{2}}{\lambda}-r+\frac{\sigma_{1}^{2}}{2 \lambda}\right) \\
& \gamma_{2}=\exp \left(\mu+\alpha \frac{\sigma_{2}^{2}}{1-\lambda}-r+\frac{\sigma_{2}^{2}}{2(1-\lambda)}\right)
\end{aligned}
$$

and :

$$
\begin{aligned}
\nu_{1} \gamma_{1} & \longrightarrow 1 \\
C_{t} & \longrightarrow 1, \quad \text { when } \quad \lambda \rightarrow 0
\end{aligned}
$$

We notice that the first component of the historical mixture distribution has a small weight whereas on the contrary it is associated with a strong weight for the risk-neutral distribution and consequently for the price of the European Call option. The first term of the price tends to 1, that is to say the (normalized) price of the underlying asset, and the second term tends to zero. This may be seen as the effect of strong kurtosis.

\subsection{The jump-diffusion model}

A general jump diffusion model, as proposed by Merton (1976), is defined as a superposition of two continuous time processes. The first one is a Brownian motion used classically to model "normal" movements on returns and the second one is constructed on the basis of a Poisson process (each Poisson event causes a normally distributed jump on returns). The latter enables to model "abnormal" movements on returns. 
Here we want to work in a discrete time context and so we define a geometric return distribution for one period. Let us first recall that the continuous time process is :

$$
\ln \left(\frac{S_{t}}{S_{0}}\right)=\mu t+\sigma B(t)+\sum_{j=1}^{N_{t}} Y_{j}
$$

where $S_{t}$ is the asset price at time $t, B(t)$ is a standard Brownian motion, $N_{t}$ is a Poisson counting process with parameter $\lambda$ and $Y_{j}$ (which measures the $j^{\text {th }}$ jump amplitude) is, for each $j \in\{1, \ldots, J\}$, independently and identically normally distributed $\mathcal{N}\left(\mu_{p}, \sigma_{p}^{2}\right)$.

The geometric return distribution on one period, i.e the distribution of $y_{t}=\log \left(S_{t} / S_{t-1}\right)$, is an infinite countable mixture of Normal distributions with Poisson weights. Its p.d.f. is the following:

$$
\sum_{j=0}^{+\infty} e^{-\lambda} \frac{\lambda^{j}}{j !} n\left(\mu+j \mu_{p}, \sigma^{2}+j \sigma_{p}^{2}\right)
$$

Therefore we are able to give analoguous formulas to (4.2), (4.3), (4.4) [see Appendix 6 for the proofs].

Proposition 9.a : The unique value of $\alpha$ is the solution of :

$$
\mu-r+\left(\alpha+\frac{1}{2}\right) \sigma^{2}+\lambda \exp \left(\alpha \mu_{p}+\sigma_{p}^{2} \frac{\alpha^{2}}{2}\right)\left[\exp \left(\mu_{p}+\sigma_{p}^{2}\left(\alpha+\frac{1}{2}\right)\right)-1\right]=0
$$

Proposition 9.b : The risk-neutral distribution is again an infinite countable mixture of Normal distributions with modified Poisson weights :

$$
f^{Q}(y)=\sum_{j=0}^{+\infty} \nu_{j} n\left(y ; \mu+\alpha \sigma^{2}+j\left(\mu_{p}+\alpha \sigma_{p}^{2}\right), \sigma^{2}+j \sigma_{p}^{2}\right)
$$

where $\nu_{j}=\exp \left(-\lambda^{\prime}\right) \frac{\lambda^{\prime j}}{j !}$ with $\lambda^{\prime}=\lambda \exp \left(\alpha \mu_{p}+\sigma_{p}^{2} \frac{\alpha^{2}}{2}\right)$.

Thus, the risk-neutral process is again of jump diffusion type, with a modified drift $\mu^{\prime}=\mu+\alpha \sigma^{2}$, the same volatility $\sigma^{2}$, a modified mean $\mu_{p}^{\prime}=\mu_{p}+\alpha \sigma_{p}^{2}$ and the same variance for the amplitude of the shock and, finally, a modified Poisson parameter $\lambda^{\prime}$.

Proposition 9.c : The price of the European Call option is an average of the Black-Scholes formulas with Poisson weights :

$$
C_{t}=\sum_{j=0}^{\infty} \beta_{j} C_{B S}\left(\sigma^{2}+j \sigma_{p}^{2}, \frac{\kappa}{\gamma_{j}}\right)
$$

where $\beta_{j}=\exp (-\tilde{\lambda}) \frac{\tilde{\lambda}^{j}}{j !}$ with $\tilde{\lambda}=\lambda \exp \left((\alpha+1) \mu_{p}+\sigma_{p}^{2} \frac{(\alpha+1)^{2}}{2}\right)$,

and $\gamma_{j}=\exp \left(\mu+j \mu_{p}+\alpha\left(\sigma^{2}+j \sigma_{p}^{2}\right)-r+\frac{\sigma^{2}+j \sigma_{p}^{2}}{2}\right)$. 


\subsection{Implied Black-Scholes volatility and historical parameters}

We have seen in previous sections that the call option pricing formula depends on parameters $\Lambda=\left(p_{j}, \mu_{j}, \sigma_{j}^{2} ; j=1, \ldots, J\right)$ of the historical distribution instead of the volatility $\sigma$ only in the BS formula. In particular, the assumption of a mixed normal distribution, able to reproduce asset's returns stylized facts such as asymmetries and fat tails, allows to describe implied volatility curves with smile and skew shapes. These features are presented on Figures 1, 2 and 3, with parameters fixed to (annualized) empirically reasonable values.

In the first case we consider a mixture of two Gaussian distributions with the same means and probabilities $\left(\mu_{1}=\mu_{2}=0.03, p=0.50\right)$ and with a global variance fixed to the level $\sigma^{2}=0.04$ : this situation allows to isolate and observe the effect of an increasing kurtosis on the implied BS volatility (for maturity one year), starting from the (flat) BS form (when $\sigma_{1}^{2}=\sigma_{2}^{2}=\sigma^{2}=0.04$ ) and applying an increasing variance in the second component of the mixture ${ }^{5}$ ( $\sigma_{1}^{2}$ contemporaneously reduces to guarantee a fixed global variance). The implied volatility induced by our model takes the smile shape when the kurtosis coefficient leaves the BS case and takes higher values. In particular, we are able to reproduce the empirically observed fact that the BS formula tends to underprice out-of-the-money and in-the-money options, while overpricing at-the-money options [see Figure 1]; moreover, the values of the implied volatilities replicated by this simple static model are close to the observed ones for European Index options [see, for instance, Pan (2002)].

In the second case, we get a more asymmetric smile by taking a mixture of two Gaussian random variables with higher means $\left(\mu_{1}=\mu_{2}=0.07\right)$ and variances than in the first case, but now the first component has a much higher weight than the second one; as in the previous case, we consider a fixed global variance $\left(\sigma^{2}=0.05\right)$ and we induce an increasing kurtosis by an increasing change in $\sigma_{2}^{2}$ ( $\sigma_{1}^{2}$ reduces to keep the global variance fixed). This structure describes a market with high expected returns and with a (typical) low volatility scenario that sometimes switches to an high volatility one : the combination of these two features by means of the mixed normal model leads to produce, at realistic values, implied BS volatilities with more asymmetric smiles [see Figure 2].

In the third case [see Figure 3], we present the volatility skew, that is, an implied volatility shape typical of equity options. Indeed, in this situation the historical distribution of the return of the underlying asset is left-asymmetric with a left tail fatter than the right one because of the higher probability of large negative returns. In order to reproduce this type of distribution, we consider a mixture where the first component has a lower mean $\left(\mu_{1}=0.01\right.$ and $\mu_{2}=0.08$, with $\left.p=0.5\right)$, but a variance higher than the second one. As in the previous cases, we consider a fixed global variance and we observe the effect of an increasing negative skewness induced by an increasing value in the variance of the first component ${ }^{6}$. Figure 3 shows that as the negative skewness increases with the size of the left tail, the implied volatility takes a more pronounced skewed shape denoting the induced higher value for out-of-the-money Put options and in-the-money Call options.

In Figure 4, we present the implied volatility surface obtained from the static Mixed-Normal model (the time-to-maturity, measured in years, changes from 0.25 to 1.5). We can observe that, as the maturity increases, the profile of implied volatility becomes flatter denoting, consequently, an increasing presence of risk-neutral normality in the distribution of the underlying return. We will see in Section 8.4 that the Switching Regime specification is able to replicate a surface closer

\footnotetext{
${ }^{5}$ It is easy to verify that, in the case of a mixture of two gaussian random variables with the same means and with a global variance $\sigma^{2}$ fixed to a certain level $\mathrm{M}, \tilde{\mu}_{3}=0$ and $\tilde{\mu}_{4}=g\left(M, \sigma_{2}^{2}\right)$ with $\left(\partial \tilde{\mu}_{4} / \partial \sigma_{2}^{2}\right) \gtreqless 0$ if and only if $\sigma_{2}^{2} \gtreqless M$.

${ }^{6}$ In this situation, the movement on $\sigma_{1}^{2}$ modifies at the same time the skewness and kurtosis parameters, but the fact to consider a fixed global variance with $\mu_{1}$ lower than $\mu_{2}$ guarantees to have $\tilde{\mu}_{3}<0$ for every $\sigma_{1}^{2}>$ $\sigma_{2}^{2}+\frac{1}{3}\left(\mu_{1}-\mu_{2}\right)^{2}(2 p-1)$ and the skewness parameter takes higher negative values when the difference between $\sigma_{1}^{2}$ and the RHS of the inequality increases.
} 
to those observed, for instance, for European-style Index options [see Cont and da Fonseca (2002)].

Now, if we consider the previous volatility skew case, without fixing the global variance, we can observe the movement of the implied BS volatility in a context characterized at the same time by increasing global variance and skewness and by changes in the kurtosis coefficient. Figure 5 shows that the variations in variance, skewness and kurtosis, induced by an increasing value of $\sigma_{1}^{2}$, gives an implied volatility curve that, starting from the BS case, takes more pronounced skewed shapes at higher value levels of volatility. In other words, in this more general case, we have at the same time level and skew effects.

It is also interesting to study the behaviour of the implied BS volatility of the extreme-risks parameterization presented in Section 4.2. Here, as $\lambda$ tends to zero, the geometric return (in the historical framework) converges in distribution to the Gaussian law, but with a kurtosis increasing to infinity (convergence in distribution without convergence of moments): Figure 6 (in which we set $\left.\sigma_{1}^{2}=\sigma_{2}^{2}\right)$ shows that starting from the flat implied volatility $(\lambda=0.5)$, if we consider a decreasing value of $\lambda$, the induced higher kurtosis leads to a smile shape as presented in Figures 1 and 2, but now, since the kurtosis tends rapidly to infinity when $\lambda$ tends to zero, the implied BS volatility increases quickly for every moneyness strike [see Figure 7] denoting the effect induced by extreme risks. Observe that the implied volatilities associated to large kurtosis take values close to those we frequently observe in an high volatility option market.

\section{The Static Nonparametric Case}

The models presented in the previous sections are based on a parametric historical distribution for stock returns. Let us now consider the Nonparametric static viewpoint, combined with a parametric pricing kernel (2.2), for option pricing. In this approach, the geometric returns are i.i.d., their distribution is not specified and is estimated by means of the well-known Gaussian kernel density estimator. It turns out that this estimator is a mixture of normal distributions with constant variances [equal to $b^{2}$, where $b$ is the smoothing parameter (bandwidth)] and that the weights are all equal.

We note $\left(y_{1}, y_{2}, \ldots, y_{J}\right)$ the observations of the geometric return. The Gaussian kernel estimator is :

$$
f(y)=\sum_{j=1}^{J} \frac{1}{J} n\left(y ; y_{j}, b^{2}\right),
$$

Therefore, by a direct application of formulas (4.2), (4.3), (4.4), we obtain the following results: First, $\alpha$ is the unique solution of :

$$
\sum_{j=1}^{J} \exp \left(\alpha y_{j}\right)\left[\exp \left(y_{j}+b^{2} \alpha+\frac{b^{2}}{2}\right)-\exp r\right]=0 .
$$

Second, the risk-neutral p.d.f. is :

$$
f^{Q}(y)=\sum_{j=1}^{J} \nu_{j} n\left(y ; y_{j}+\alpha b^{2}, b^{2}\right)
$$

where, for $j=1, \ldots, J$

$$
\nu_{j}=\frac{\exp \left(\alpha y_{j}\right)}{\sum_{j=1}^{J} \exp \left(\alpha y_{j}\right)} .
$$


Third, the price of the European Call option is :

$$
C_{t}=\sum_{j=1}^{J} \nu_{j}^{\prime} C_{B S}\left(b^{2}, \frac{\kappa}{\gamma_{j}}\right),
$$

with

$$
\nu_{j}^{\prime}=\frac{\exp \left[(\alpha+1) y_{j}\right]}{\sum_{j=1}^{J} \exp \left[(\alpha+1) y_{j}\right]}
$$

and

$$
\gamma_{j}=\exp \left(y_{j}+\alpha b^{2}-r+\frac{b^{2}}{2}\right)
$$

Thus, using the Gaussian kernel estimator, it is possible to include the general Nonparametric case in the framework of normal mixtures, and to derive general procedures to obtain risk-neutral distributions and option pricing formulas ${ }^{7}$.

\section{The Mixed-Normal GARCH Model}

The aim of this section and the following ones is to extend the model presented in Section 4.1 to a dynamic framework. In this section, the dynamics is introduced by means of a GARCH-type characterization of the geometric stock return with a conditional distribution defined by a Mixture of Gaussian random variables. In Section 7 we present a Dynamic Semi-Parametric specification for the historical dynamics of the log return, while Section 8 deals with the Switching Regime case.

The Mixed-Normal GARCH model presented here gives the possibility to describe in a simple and realistic way not only the typical stylized facts of asset returns as volatility clustering, heavy tails and asymmetries, but also new emerging behaviours like time-varying skewness and kurtosis as indicated in the papers of Hansen (1994), Paolella (1999), Harvey and Siddique (1999), Brännäs and Nordman (2001), Rockinger and Jondeau $(2002)^{8}$.

Let us first consider a mixture of two Gaussian distributions with a mean equal to zero, which will be used to derive classes of martingale difference sequences. Such a distribution can be parameterized as :

$$
f(u)=\lambda n\left(u ; a(1-\lambda), \sigma_{1}^{2}\right)+(1-\lambda) n\left(u ;-a \lambda, \sigma_{2}^{2}\right)
$$

where $0 \leq \lambda \leq 1$, and $a \in \mathbb{R}$.

\footnotetext{
${ }^{7}$ Ait-Sahalia and Duarte (2003) also proposed a non parametric option pricing methodology where, working directly in the risk-neutral setting, they estimate the state price density from a cross-section of option prices. Their approach needs to impose shape restrictions on the pricing functions in order to satisfy the absence of arbitrage conditions [the price of a Call option must be a decreasing and convex function of the strike]. Our Mixed-Normal Nonparametric approach, based on the exponential-affine SDF change of measure, automatically guarantee the absence of arbitrage restrictions and, actually, the pricing function (5.4) is given by a linear combination of BlackScholes formulas.

${ }^{8}$ Camara (2003) and Christoffersen, Heston and Jacobs (2004) present option pricing models, with GARCH volatility dynamics, under alternative distributional assumptions.
} 
The mean, variance, skewness and kurtosis are

$$
\begin{aligned}
\mu= & 0 \\
\sigma^{2}= & {\left[\lambda \sigma_{1}^{2}+(1-\lambda) \sigma_{2}^{2}\right]+a^{2} \lambda(1-\lambda) } \\
\tilde{\mu}_{3}= & \frac{1}{\sigma^{3}}\left\{a \lambda(1-\lambda)\left[3\left(\sigma_{1}^{2}-\sigma_{2}^{2}\right)\right]\right\} \\
\tilde{\mu}_{4}= & \frac{1}{\sigma^{4}}\left\{3\left[\lambda \sigma_{1}^{4}+(1-\lambda) \sigma_{2}^{4}\right]+6 a^{2} \lambda(1-\lambda)\left[(1-\lambda) \sigma_{1}^{2}+\lambda \sigma_{2}^{2}\right]\right. \\
& \left.\quad+a^{4} \lambda(1-\lambda)\left[3 \lambda^{2}-3 \lambda+1\right]\right\} .
\end{aligned}
$$

Normal mixtures in a GARCH context were suggested by Vlaar and Palm (1993, 1997), Bauwens et al. (1999) and Lin and Yeh (2000). All these modelisations are special cases of the general specification proposed by Hass, Mittnick and Paolella (2002)[HMP hereafter].

The GARCH characterizations of the dynamics we propose for our pricing framework are of two types, and the first one is a particular case of the HMP model [see Appendix 7]. In both cases, the binary process $z_{t}$, giving the Gaussian component, is an exogenous white noise and, therefore, conditionally to its own past, $y_{t}$ follows a mixture of normal distributions.

\subsection{The MN-GARCH process of first type}

In this case, the conditional distribution of process $\left(\varepsilon_{t}\right)$ is a mixture of two Gaussian distributions :

$$
f\left(\varepsilon_{t+1} \mid \underline{\varepsilon_{t}}\right)=\lambda n\left(\varepsilon_{t+1} ; a(1-\lambda), \sigma_{1 t+1}^{2}\right)+(1-\lambda) n\left(\varepsilon_{t+1} ;-a \lambda, \sigma_{2 t+1}^{2}\right),
$$

where $\varepsilon_{t}:=\left(\varepsilon_{t}, \varepsilon_{t-1}, \ldots\right)$ is the information on the current and lagged values of $\varepsilon_{t}$ and where the variances of the mixture components evolve according to GARCH specifications. In order to keep the notation simple, we consider $\operatorname{GARCH}(1,1)$ specifications (a generalization to $\operatorname{GARCH}(p, q$ ) structures is straightforward) :

$$
\left\{\begin{array}{l}
\sigma_{1 t+1}^{2}=\omega_{1}+b_{1} \varepsilon_{t}^{2}+c_{1} \sigma_{1 t}^{2} \\
\sigma_{2 t+1}^{2}=\omega_{2}+b_{2} \varepsilon_{t}^{2}+c_{2} \sigma_{2 t}^{2},
\end{array}\right.
$$

submitted to non-negativity conditions $c_{i} \geq 0, \omega_{i}>0$ and $b_{i} \geq 0, i \in\{1,2\}$.

Conditionally to the values of an i.i.d. latent process giving the chosen component at each date $t$, the process is gaussian conditionally to its past. The process $\varepsilon_{t}$ is a martingale difference sequence, since $E\left(\varepsilon_{t+1} \mid \underline{\varepsilon_{t}}\right)=0$, and can be used as a building block for more complex models, such as ARMA-GARCH or GARCH-M models.

It is possible to verify [see Appendix 6] that $\varepsilon_{t}$ also has a GARCH specification, if we impose the constraint $c_{1}=c_{2}=c$. Indeed, in this case, we can write :

$$
E\left(\varepsilon_{t+1}^{2} \mid \underline{\varepsilon_{t}}\right):=\sigma_{t+1}^{2}=\xi+\left[\lambda b_{1}+(1-\lambda) b_{2}\right] \varepsilon_{t}^{2}+c \sigma_{t}^{2},
$$

with $\xi:=(1-c) a^{2} \lambda(1-\lambda)+\lambda \omega_{1}+(1-\lambda) \omega_{2}$. 
In this type of model, the conditional distribution of the standardized variable $\frac{\varepsilon_{t+1}}{\sigma_{t+1}}$, given $\varepsilon_{t}$, is a mixture of normal distributions depending on the past, and a conditional Maximum Likelihood estimation procedure can be followed, while the second type of MN-GARCH model presented below gives an i.i.d. specification to the standardized process and a useful two-step estimation procedure is proposed.

\subsection{The MN-GARCH process of second type}

If we consider again the simple $\operatorname{GARCH}(1,1)$ specification, the model takes the following form :

$$
\left\{\begin{array}{l}
\varepsilon_{t+1}=\sigma_{t+1} u_{t+1} \\
\sigma_{t+1}^{2}=\omega+c \varepsilon_{t}^{2}+d \sigma_{t}^{2},
\end{array}\right.
$$

where $\left(u_{t}\right)$ is a sequence of independent zero mean mixed-normal random variables characterized by the following p.d.f. :

$$
f\left(u_{t}\right)=\lambda n\left(u_{t} ; a(1-\lambda), \sigma_{1}^{2}\right)+(1-\lambda) n\left(u_{t} ;-a \lambda, \sigma_{2}^{2}\right),
$$

and where the conditional p.d.f. of $\varepsilon_{t}$ takes the following form :

$$
f\left(\varepsilon_{t+1} \mid \underline{\varepsilon_{t}}\right)=\lambda n\left(\varepsilon_{t+1} ; \sigma_{t+1} a(1-\lambda), \sigma_{t+1}^{2} \sigma_{1}^{2}\right)+(1-\lambda) n\left(\varepsilon_{t+1} ;-\sigma_{t+1} a \lambda, \sigma_{t+1}^{2} \sigma_{2}^{2}\right) .
$$

Thus, conditionally to the i.i.d. latent process giving the chosen component at each date, the process is also conditionally Gaussian. By definition, the variables $\frac{\varepsilon_{t}}{\sigma_{t}}$ are i.i.d.. Note that the model is overparameterized, and in order to solve the identification problem, we propose two possible identification restrictions.

\section{i) Normalization of the variance of $u_{t}$}

This first restriction imposes that :

$$
V\left(u_{t}\right)=\left[\lambda \sigma_{1}^{2}+(1-\lambda) \sigma_{2}^{2}\right]+a^{2} \lambda(1-\lambda)=1,
$$

and, consequently, we obtain $V\left(\varepsilon_{t+1} \mid \underline{\varepsilon_{t}}\right) \equiv \sigma_{t+1}^{2}$ and $V\left(\varepsilon_{t+1}\right) \equiv E\left(\sigma_{t+1}^{2}\right)$.

ii) Normalization of $E\left(\sigma_{t+1}^{2}\right)$

In this second case, we impose :

$$
E\left(\sigma_{t+1}^{2}\right)=\frac{\omega}{1-c-d}=1
$$

or $\omega=1-c-d$. So $V\left(\varepsilon_{t+1} \mid \underline{\varepsilon_{t}}\right)=\sigma^{2} \sigma_{t+1}^{2}$ where $\sigma^{2}=V\left(u_{t+1}\right)=V\left(\varepsilon_{t+1}\right)$.

The advantage of this modelisation is the possibility to implement a two-step estimation procedure for the model parameters.

\section{a) First step :}

We estimate the variance $\sigma^{2}$ of the marginal distribution of $\left(\varepsilon_{t}\right)$ by the empirical variance $\hat{\sigma}^{2}$ and the parameter $\theta_{1}=(c, d)$ by a Pseudo-Maximum Likelihood procedure (based on a Gaussian GARCH model) applied to $\left(\frac{\varepsilon_{t}}{\hat{\sigma}}\right)$ and using the restriction $\omega=1-c-d$. 


\section{b) Second step :}

From the estimated values $\hat{\sigma}_{t}$ we get an estimated sequence of independent mixed-normal

random variables $\left(\frac{\varepsilon_{t}}{\tilde{\sigma}_{t}}\right)$ for which we proceed to the estimation of the mixture parameters $\theta_{2}=$ $\left(\lambda, a, \sigma_{1}^{2}, \sigma_{2}^{2}\right)$. We can use, for instance, a Maximum Likelihood estimation or a Bayesian Approach estimation using posterior simulation via Monte Carlo Markov Chain (MCMC) methods [see McLachlan and Peel (2000) and the references therein].

An advantage of the first type of normalization is that it allows for an IGARCH modelisation of $\left(\varepsilon_{t+1} \mid \underline{\varepsilon_{t}}\right)$, whereas the second normalization condition makes (by construction) impossible this specification.

\subsection{Computation of the SDF and of the risk-neutral distribution}

With the definition of the conditional distribution of the stock return $y_{t+1}=\varepsilon_{t+1}{ }^{9}$, we have the dynamic structure that gives us the possibility to specify for every date $t$ (conditionally to $I_{t}=\underline{\varepsilon_{t}}$ ) the pricing model presented in Section 4.1.1. More precisely, we can derive the following results about the SDF and the risk-neutral distribution.

Proposition 10 : If the historical distribution of the process $y_{t}$ is a MN-GARCH of first or second type and if the stochastic discount factor is exponential-affine, we have for every date $t$ a unique solution $\alpha_{t}=\alpha\left(I_{t}\right)$ and $\beta_{t}=\beta\left(I_{t}\right)$ that satisfies system (2.3). The unique value of $\alpha_{t}$ is solution of :

$$
\begin{aligned}
& \lambda \exp \left[\alpha_{t} a(1-\lambda)+\sigma_{1 t}^{2} \frac{\alpha_{t}^{2}}{2}\right]\left[\exp \left(a(1-\lambda)+\sigma_{1 t}^{2} \alpha_{t}+\frac{\sigma_{1 t}^{2}}{2}\right)-\exp r_{t+1}^{f}\right] \\
& +(1-\lambda) \exp \left[\sigma_{2 t}^{2} \frac{\alpha_{t}^{2}}{2}-\alpha_{t} a \lambda\right]\left[\exp \left(\sigma_{2 t}^{2} \alpha_{t}+\frac{\sigma_{2 t}^{2}}{2}-a \lambda\right)-\exp r_{t+1}^{f}\right]=0
\end{aligned}
$$

for the MN-GARCH of first type, while, if we consider the MN-GARCH of second type, the unique value of $\alpha_{t}$ is solution of :

$$
\begin{aligned}
& \lambda \exp \left[\sigma_{t} \alpha_{t}\left(a(1-\lambda)+\sigma_{1}^{2} \frac{\sigma_{t} \alpha_{t}}{2}\right)\right]\left[\exp \left(\sigma_{t} a(1-\lambda)+\sigma_{t}^{2} \sigma_{1}^{2} \alpha_{t}+\frac{\sigma_{t}^{2} \sigma_{1}^{2}}{2}\right)-\exp r_{t+1}^{f}\right] \\
& +(1-\lambda) \exp \left[\sigma_{t} \alpha_{t}\left(\sigma_{2}^{2} \frac{\sigma_{t} \alpha_{t}}{2}-a \lambda\right)\right]\left[\exp \left(\sigma_{t}^{2} \sigma_{2}^{2} \alpha_{t}+\frac{\sigma_{t}^{2} \sigma_{2}^{2}}{2}-\sigma_{t} a \lambda\right)-\exp r_{t+1}^{f}\right]=0 .
\end{aligned}
$$

Given the unique solution $\left(\alpha_{t}, \beta_{t}\right)$, we can compute for every $t$ the unique SDF $M_{t, t+1}$ and, consequently, the unique mixed-normal conditional risk-neutral distribution.

Proposition 11 : The conditional risk-neutral distribution of the MN-GARCH process is unique and is a mixture of Normal distributions. For the MN-GARCH process of first type the risk-neutral p.d.f. is given by :

$$
f_{1}^{Q}\left(y_{t+1}\right)=\nu_{1 t} n\left(y_{t+1} ; a(1-\lambda)+\alpha_{t} \sigma_{1 t}^{2}, \sigma_{1 t}^{2}\right)+\left(1-\nu_{1 t}\right) n\left(y_{t+1} ; \alpha_{t} \sigma_{2 t}^{2}-a \lambda, \sigma_{2 t}^{2}\right),
$$

\footnotetext{
${ }^{9}$ The modelisation of the stock return dynamics can be obviously generalized by the definition of a dynamic statistical model (for instance, ARMA model) for $y_{t+1}$ in which the mixed-normal process $\varepsilon_{t+1}$ is introduced as the noise component.
} 
where $0 \leq \nu_{1 t} \leq 1$ and

$$
\nu_{1 t}=\frac{\lambda \exp \left(\alpha_{t} a(1-\lambda)+\sigma_{1 t}^{2} \frac{\alpha_{t}^{2}}{2}\right)}{\lambda \exp \left(\alpha_{t} a(1-\lambda)+\sigma_{1 t}^{2} \frac{\alpha_{t}^{2}}{2}\right)+(1-\lambda) \exp \left(\sigma_{2 t}^{2} \frac{\alpha_{t}^{2}}{2}-\alpha_{t} a \lambda\right)},
$$

while, for the MN-GARCH process of second type the risk-neutral p.d.f. is the following :

$f_{2}^{Q}\left(y_{t+1}\right)=\nu_{2 t} n\left(y_{t+1} ; \sigma_{t}\left(a(1-\lambda)+\alpha_{t} \sigma_{t} \sigma_{1}^{2}\right), \sigma_{t}^{2} \sigma_{1}^{2}\right)+\left(1-\nu_{2 t}\right) n\left(y_{t+1} ; \sigma_{t}\left(\alpha_{t} \sigma_{t} \sigma_{2}^{2}-a \lambda\right), \sigma_{t}^{2} \sigma_{2}^{2}\right)$,

where $0 \leq \nu_{2 t} \leq 1$ and

$$
\nu_{2 t}=\frac{\lambda \exp \left[\sigma_{t} \alpha_{t}\left(a(1-\lambda)+\sigma_{t} \sigma_{1}^{2} \frac{\alpha_{t}}{2}\right)\right]}{\lambda \exp \left[\sigma_{t} \alpha_{t}\left(a(1-\lambda)+\sigma_{t} \sigma_{1}^{2} \frac{\alpha_{t}}{2}\right)\right]+(1-\lambda) \exp \left[\sigma_{t} \alpha_{t}\left(\sigma_{t} \sigma_{2}^{2} \frac{\alpha_{t}}{2}-a \lambda\right)\right]} .
$$

\subsection{Derivative Pricing}

With the specification of the (unique) risk-neutral probability distribution for the one-period stock return $y_{t+1}$ (conditionally to $I_{t}$ ), we can specify the following option pricing formulas ${ }^{10}$.

Proposition 12 : The price at the date $t$ of the European Call option with maturity one and payoff $\left(\exp y_{t+1}-\kappa_{t}\right)^{+}$is, for the MN-GARCH process of the first type :

$$
C_{1 t}=\nu_{1 t} \gamma_{1 t, 1} C_{B S}\left(\sigma_{1 t}^{2}, \frac{\kappa_{t}}{\gamma_{1 t, 1}}\right)+\left(1-\nu_{1 t}\right) \gamma_{1 t, 2} C_{B S}\left(\sigma_{2 t}^{2}, \frac{\kappa_{t}}{\gamma_{1 t, 2}}\right)
$$

where the (one-period) Black-Scholes formula is defined for a volatility $\sigma_{j t}^{2}$ and a moneyness strike $\kappa_{t} / \gamma_{1 t, j}, j \in\{1,2\}$, and

$$
\begin{aligned}
\gamma_{1 t, 1} & =\exp \left[\alpha_{t} \sigma_{1 t}^{2}+\frac{\sigma_{1 t}^{2}}{2}+a(1-\lambda)-r_{t+1}^{f}\right] \\
\gamma_{1 t, 2} & =\exp \left[\alpha_{t} \sigma_{2 t}^{2}+\frac{\sigma_{2 t}^{2}}{2}-a \lambda-r_{t+1}^{f}\right] .
\end{aligned}
$$

Moreover, by the procedure given in Appendix 4, it can be shown that :

$$
\begin{aligned}
& 0 \leq \nu_{1 t} \gamma_{1 t, 1} \leq 1, \quad 0 \leq\left(1-\nu_{1 t}\right) \gamma_{1 t, 2} \leq 1 \\
& \nu_{1 t} \gamma_{1 t, 1}+\left(1-\nu_{1 t}\right) \gamma_{1 t, 2}=1, \forall t>0 .
\end{aligned}
$$

If we consider the MN-GARCH process of the second type, the pricing formula is given by :

$$
C_{2 t}=\nu_{2 t} \gamma_{2 t, 1} C_{B S}\left(\sigma_{t}^{2} \sigma_{1}^{2}, \frac{\kappa_{t}}{\gamma_{2 t, 1}}\right)+\left(1-\nu_{2 t}\right) \gamma_{2 t, 2} C_{B S}\left(\sigma_{t}^{2} \sigma_{2}^{2}, \frac{\kappa_{t}}{\gamma_{2 t, 2}}\right),
$$

\footnotetext{
${ }^{10}$ In the literature, the well known GARCH option pricing models of Duan (1995) and Heston and Nandi (2002) are particular cases of our approach given that they consider Gaussian $y_{t+1}$, conditionally to its own past. Moreover, they consider a constant market price of risk $\alpha_{t}=\alpha$. Also Christoffersen, Elkahmi and Jacobs (2005) consider a conditional Gaussian distribution for the log return, but with the (time-varying) conditional mean and variance restricted to be predetermined processes.
} 
where the (one-period) Black-Scholes formula is now defined for a volatility $\sigma_{t}^{2} \sigma_{j}^{2}$ and a moneyness strike $\kappa_{t} / \gamma_{2 t, j}, j \in\{1,2\}$, and

$$
\begin{aligned}
\gamma_{2 t, 1} & =\exp \left[\alpha_{t} \sigma_{t}^{2} \sigma_{1}^{2}+\frac{\sigma_{t}^{2} \sigma_{1}^{2}}{2}+\sigma_{t} a(1-\lambda)-r_{t+1}^{f}\right] \\
\gamma_{2 t, 2} & =\exp \left[\alpha_{t} \sigma_{t}^{2} \sigma_{2}^{2}+\frac{\sigma_{t}^{2} \sigma_{2}^{2}}{2}-\sigma_{t} a \lambda-r_{t+1}^{f}\right] .
\end{aligned}
$$

In addition, we still have that :

$$
\begin{aligned}
& 0 \leq \nu_{2 t} \gamma_{2 t, 1} \leq 1, \quad 0 \leq\left(1-\nu_{2 t}\right) \gamma_{2 t, 2} \leq 1 \\
& \nu_{2 t} \gamma_{2 t, 1}+\left(1-\nu_{2 t}\right) \gamma_{2 t, 2}=1, \forall t>0
\end{aligned}
$$

The generalization of the propositions above to the case of a mixture of $J$ components is straightforward.

\section{The Dynamic Semi-Parametric Case}

In this section, we develop a semi-parametric analysis of the Mixed Normal dynamic pricing model proposed in the previous sections. In particular, we consider that the geometric return satisfies :

$$
y_{t+1}=m_{t+1}+\sigma_{t+1} \varepsilon_{t+1}, \sigma_{t+1}>0
$$

where $m_{t}$ and $\sigma_{t}$ are the location and scale parameters, respectively, that may depend on lagged values of the return, and $\left(\varepsilon_{t}\right)$ is a sequence of i.i.d. variables.

This approach is very similar to the one proposed by Gourieroux and Monfort (2006), which is based on the estimated empirical distribution of the errors $\left(\varepsilon_{t}\right)$. Minimal assumptions are made about the log-return dynamic process, and for this reason the methodology presented here appears as a promising tool for option pricing ${ }^{11}$. In particular, we consider a parametric specification of $m_{t}$ and $\sigma_{t}$ :

$$
m_{t}=m\left(y_{t-1} ; \theta\right), \sigma_{t}=\sigma\left(y_{t-1} ; \theta\right),
$$

and we leave unspecified the distribution of the error term. The available observations on the returns are denoted by $y_{1}, \ldots, y_{T}$.

The parameter $\theta$ can be consistently estimated from historical data by applying a PseudoMaximum Likelihood method. The estimator is given by :

$$
\hat{\theta}_{T}=\arg \max _{\theta} \sum_{t=1}^{T}\left\{-\log \sigma^{2}\left(y_{t-1} ; \theta\right)-\frac{\left[y_{t}-m\left(y_{t-1} ; \theta\right)\right]^{2}}{\sigma^{2}\left(y_{t-1} ; \theta\right)}\right\} ;
$$

then we compute the residuals:

$$
\hat{\varepsilon}_{\tau}=\frac{y_{\tau}-m\left(y_{\tau-1} ; \hat{\theta}_{T}\right)}{\sigma\left(y_{\tau-1} ; \hat{\theta}_{T}\right)}, \tau=2, \ldots, T
$$

\footnotetext{
${ }^{11}$ Ait-Sahalia and Lo (1998) follow a semi-parametric approach in which the non parametric deterministic volatility function is an input in the Black-Scholes formula. Duan (2002), following the relative entropy approach of Stutzer (1996), proposes a nonparametric option pricing model, where the log-return has a conditional (historical and riskneutral) Gaussian distribution.
} 
and the distribution of $\varepsilon_{t}$ can be approximated by a Gaussian Kernel estimator based on the $\hat{\varepsilon}_{\tau}$ 's.

Therefore, the p.d.f. of $\varepsilon_{t+1}$ is approximated by the Gaussian mixture :

$$
\frac{1}{T-1} \sum_{\tau=2}^{T} n\left(\varepsilon ; \hat{\varepsilon}_{\tau}, b^{2}\right)
$$

where $b$ is the bandwidth.

The conditional historical p.d.f. of $y_{t+1}$ given $y_{t}$ is approximated by the Gaussian mixture :

$$
\frac{1}{T-1} \sum_{\tau=2}^{T} n\left(y_{t+1} ; \hat{m}_{t+1}+\hat{\sigma}_{t+1} \hat{\varepsilon}_{\tau}, \hat{\sigma}_{t+1}^{2} b^{2}\right)
$$

where $\hat{m}_{t+1}=m\left(y_{t}, \hat{\theta}_{T}\right), \hat{\sigma}_{t+1}=\sigma\left(y_{t}, \hat{\theta}_{T}\right)$.

This result allows to derive the Dynamic Semi-Parametric Mixed Normal pricing model. In particular, we obtain the following results.

Proposition 13 : If the conditional historical distribution of $y_{t+1}$ is approximated by a mixture of $(T-1)$ Normal distributions $\mathcal{M N}\left(T-1, \frac{1}{T-1}, \hat{m}_{t+1}+\hat{\sigma}_{t+1} \hat{\varepsilon}_{\tau}, \hat{\sigma}_{t+1}^{2} b^{2}\right)$ and if the stochastic discount factor is exponential-affine, we have for every $t$ a unique solution $\alpha_{t}=\alpha\left(I_{t}\right)$ and $\beta_{t}=\beta\left(I_{t}\right)$ that satisfies the system (2.3). The unique value of $\alpha_{t}$ is solution of :

$$
\begin{aligned}
\sum_{\tau=2}^{T} \exp \left[\alpha_{t}\left(\hat{m}_{t+1}+\hat{\sigma}_{t+1} \hat{\varepsilon}_{\tau}\right)+\hat{\sigma}_{t+1}^{2} b^{2} \frac{\alpha_{t}^{2}}{2}\right] \\
\quad\left[\exp \left(\left(\hat{m}_{t+1}+\hat{\sigma}_{t+1} \hat{\varepsilon}_{\tau}\right)+\hat{\sigma}_{t+1}^{2} b^{2} \alpha_{t}+\frac{\hat{\sigma}_{t+1}^{2} b^{2}}{2}\right)-\exp r_{t+1}^{f}\right]=0 .
\end{aligned}
$$

Proposition 14 : The conditional risk-neutral distribution is unique and is approximated by a mixture of Normal distributions with the following p.d.f. :

$$
f^{Q}\left(y_{t+1}\right)=\sum_{\tau=2}^{T} \nu_{\tau} n\left(y_{t+1} ; \hat{m}_{t+1}+\hat{\sigma}_{t+1}\left(\hat{\varepsilon}_{\tau}+\alpha_{t} \hat{\sigma}_{t+1} b^{2}\right), \hat{\sigma}_{t+1}^{2} b^{2}\right)
$$

where, for $\tau=2, \ldots, T$

$$
\begin{aligned}
& \nu_{\tau}=\frac{\exp \left[\alpha_{t}\left(\hat{m}_{t+1}+\hat{\sigma}_{t+1} \hat{\varepsilon}_{\tau}\right)+\hat{\sigma}_{t+1}^{2} b^{2} \frac{\alpha_{t}^{2}}{2}\right]}{\sum_{\tau=2}^{T} \exp \left[\alpha_{t}\left(\hat{m}_{t+1}+\hat{\sigma}_{t+1} \hat{\varepsilon}_{\tau}\right)+\hat{\sigma}_{t+1}^{2} b^{2} \frac{\alpha_{t}^{2}}{2}\right]}, \\
& 0 \leq \nu_{\tau} \leq 1, \sum_{\tau=2}^{T} \nu_{\tau}=1 .
\end{aligned}
$$

The risk-neutral distribution depends, for every $t$ on the estimated location and scale parameters, on the computed residuals and on the smoothing parameter $b$. 
Proposition 15 : The price of the European Call option written on exp $y_{t+1}$ with maturity one and payoff $\left(\exp y_{t+1}-\kappa\right)^{+}$is :

$$
\begin{aligned}
C_{t} & =\exp \left(-r_{t+1}^{f}\right) E^{Q}\left[\exp y_{t+1}-\kappa_{t}\right]^{+} \\
& =\sum_{\tau=2}^{T} \nu_{\tau} \gamma_{\tau} C_{B S}\left(\hat{\sigma}_{t+1}^{2} b^{2}, \frac{\kappa_{t}}{\gamma_{\tau}}\right),
\end{aligned}
$$

where $C_{B S}$ is the (one-period) Black-Scholes formula with a volatility equal to $\hat{\sigma}_{t+1}^{2} b^{2}$ and moneyness strike equal to $\kappa_{t} / \gamma_{\tau}$, and

$$
\gamma_{\tau}=\exp \left[\hat{m}_{t+1}+\hat{\sigma}_{t+1} \hat{\varepsilon}_{\tau}-r_{t+1}^{f}+\alpha_{t} \hat{\sigma}_{t+1}^{2} b^{2}+\frac{\hat{\sigma}_{t+1}^{2} b^{2}}{2}\right] .
$$

By the same procedure as in Appendix 4, it can be shown that:

$$
0 \leq \nu_{\tau} \gamma_{\tau} \leq 1, \sum_{\tau=2}^{T} \nu_{\tau} \gamma_{\tau}=1
$$

The three above propositions show the flexibility of the Mixed-Normal framework, combined with exponential-affine SDF, for a general option pricing procedure. In particular, the MixedNormal nature of the Gaussian kernel estimation allows, by the application of the results presented above, to specify a general Dynamic Semi-Parametric pricing model, starting from minimal assumptions about the stock return historical dynamics.

\section{The Switching Regimes Option Pricing Model}

\subsection{The General Switching Regimes pricing formula}

In this Section, we extend the definition of i.i.d. mixtures of conditionally normal processes to the case of regime switching models. For this purpose, we allow the unobservable process $z_{t}$, which governs at every date $t$ the choice of the component of the Gaussian mixture, to depend on its past (and possibly on $\underline{y_{t-1}}$ ).

In the sequel, we will assume that the process $z_{t}$ can take $J$ values which will be identified to $e_{j}=[0 \ldots 1 \ldots 0]^{\prime}, j=1, \ldots, J$, the vector whose components are zeros except the $j^{\text {th }}$ one that is equal to one if $j$ is the current regime.

In addition, we consider that the information available at date $t$ for the investor is given by $I_{t}=\left(y_{t}, \underline{z}_{t}\right)=\left(y_{t}, z_{t}, y_{t-1}, z_{t-1}, \ldots\right)$, that is, we assume that the investor observes the returns and the regimes. Then, we choose an exponential-affine SDF :

$$
M_{t, t+1}=\exp \left[\alpha_{t} y_{t+1}+\delta_{t}^{\prime} z_{t+1}+\beta_{t}\right]
$$

where the coefficients $\alpha_{t}, \beta_{t}$ and $\delta_{t}=\left[\delta_{1 t}, \ldots, \delta_{J t}\right]^{\prime}$ are functions of $I_{t}$. This parametric specification of the SDF gives the possibility to price separately the risk associated with the stock return dynamics and those coming from the switching of regimes. In this way, the state dependent pricing kernel gives the possibility to study how the different risk factors affect option prices, leading to a more precise knowledge of the risk attitude of investors towards different sources of risk [see, among the others, Gordon and St-Amour (2000), Melino and Yang (2003), Calvet and Fisher (2004) for details about state dependent stochastic discount factors]. Note that, for identification reasons, we can always assume $\beta_{t}=0$. 
Let us now point out an important difference with the previous sections. The pricing formula for the riskfree asset and the risky asset still gives two arbitrage free conditions that induce restrictions on the risk correction coefficients $\alpha_{t}$ and $\delta_{t}$, but it does not suffice to uniquely determine the SDF. Indeed, denoting by $\varphi_{t}(u, v)$ the conditional Laplace transform of $\left(y_{t+1}, z_{t+1}\right)$, given $I_{t}$, the constraints are :

$$
\left\{\begin{array}{l}
\exp \left(r_{t+1}^{f}\right) \varphi_{t}\left(\alpha_{t}, \delta_{t}\right)=1 \\
\varphi_{t}\left(\alpha_{t}+1, \delta_{t}\right)=1
\end{array}\right.
$$

and we get two equations for $J+1$ unknowns.

The $\alpha_{t}=\alpha\left(\underline{y_{t}}, \underline{z_{t}}\right), \delta_{t}=\delta\left(\underline{y_{t}}, \underline{z_{t}}\right)$ might be specified parametrically and estimated from a calibration of the derivative prices obtained below.

The conditional p.d.f. of the process $\left(y_{t+1}, z_{t+1}\right)$, given $I_{t}$, can be written as :

$$
f\left(y_{t+1}, z_{t+1} \mid \underline{y_{t}}, \underline{z_{t}}\right)=f\left(y_{t+1} \mid \underline{y_{t}}, \underline{z_{t+1}}\right) \times f\left(z_{t+1} \mid \underline{y_{t}}, \underline{z_{t}}\right),
$$

and we assume that the distribution of $y_{t+1}$ given $\left(\underline{y_{t}}, \underline{z_{t+1}}\right)$ is Gaussian with mean and variance respectively denoted $\mu\left(\underline{y_{t}}, \underline{z_{t+1}}\right)$ and $\sigma^{2}\left(\underline{y_{t}}, \underline{z_{t+1}}\right)$.

Proposition 16 : The Laplace transform $\varphi_{t}(u, v)$ of $\left(y_{t+1}, z_{t+1}\right)$, given $I_{t}$, is given by :

$$
\begin{aligned}
\varphi_{t}(u, v) & =E\left[\exp \left(u y_{t+1}+v^{\prime} z_{t+1}\right) \mid \underline{y_{t}}, \underline{z_{t}}\right] \\
& =\sum_{z_{t+1}} p_{t}\left(z_{t+1}\right) \exp \left(v^{\prime} z_{t+1}+u \mu\left(\underline{y_{t}}, \underline{z_{t+1}}\right)+\frac{1}{2} u^{2} \sigma^{2}\left(\underline{y_{t}}, \underline{z_{t+1}}\right)\right),
\end{aligned}
$$

where $p_{t}\left(z_{t+1}\right)$ is another notation for the conditional probability $f\left(z_{t+1} \mid \underline{y_{t}}, \underline{z_{t}}\right)$. [Proof : see Appendix 8].

In particular, the distribution of $\left(y_{t+1}\right)$, given $\left(\underline{y_{t}}, \underline{z_{t}}\right)$, is a mixture of the Gaussian distributions $\mathcal{N}\left(\mu\left(\underline{y_{t}}, \underline{z_{t+1}}\right), \sigma^{2}\left(\underline{y_{t}}, \underline{z_{t+1}}\right)\right)$.

Proposition 17 : The conditional risk-neutral distribution $Q_{t}$ of $\left(y_{t+1}, z_{t+1}\right)$, given $I_{t}$, has a Laplace transform given by :

$$
\begin{aligned}
\varphi^{Q_{t}}(u, v) & =E^{Q_{t}}\left[\exp \left(u y_{t+1}+v^{\prime} z_{t+1}\right) \mid \underline{y_{t}}, \underline{z_{t}}\right] \\
& =\sum_{z_{t+1}} \nu_{t}\left(z_{t+1}\right) \exp \left[v^{\prime} z_{t+1}+u\left[\mu\left(\underline{y_{t}}, \underline{z_{t+1}}\right)+\alpha_{t} \sigma^{2}\left(\underline{y_{t}}, \underline{z_{t+1}}\right)\right]+\frac{1}{2} u^{2} \sigma^{2}\left(\underline{y_{t}}, \underline{z_{t+1}}\right)\right]
\end{aligned}
$$

where

$$
\nu_{t}\left(z_{t+1}\right)=\frac{p_{t}\left(z_{t+1}\right) \exp \left(\delta_{t}^{\prime} z_{t+1}+\alpha_{t} \mu\left(\underline{y_{t}}, \underline{z_{t+1}}\right)+\frac{1}{2} \alpha_{t}^{2} \sigma^{2}\left(\underline{y_{t}}, \underline{z_{t+1}}\right)\right)}{\sum_{z_{t+1}} p_{t}\left(z_{t+1}\right) \exp \left(\delta_{t}^{\prime} z_{t+1}+\alpha_{t} \mu\left(\underline{y_{t}}, \underline{z_{t+1}}\right)+\frac{1}{2} \alpha_{t}^{2} \sigma^{2}\left(\underline{y_{t}}, \underline{z_{t+1}}\right)\right)} .
$$

[Proof : see Appendix 8]. 
Therefore, in the risk-neutral world, the process $\left(y_{t+1}, z_{t+1}\right)$ has exactly the same structure as in the historical world, with modified weights $\nu_{t}\left(z_{t+1}\right)$ and means for the components of the distribution of $\left(y_{t+1}\right)$ equal to $\mu\left(\underline{y_{t}}, \underline{z_{t+1}}\right)+\alpha_{t} \sigma^{2}\left(\underline{y_{t}}, \underline{z_{t+1}}\right)$.

Proposition 18 : The price at date $t$ of the European Call option with maturity one and payoff $\left(\exp y_{t+1}-\kappa_{t}\right)^{+}$, is given by the following formula ${ }^{12}$ :

$$
\begin{aligned}
C_{t} & =\exp \left(-r_{t+1}^{f}\right) E^{Q_{t}}\left[\exp y_{t+1}-\kappa_{t}\right]^{+} \\
& =\sum_{z_{t+1}} \nu_{t}\left(z_{t+1}\right) \gamma_{t}\left(z_{t+1}\right) C_{B S}\left(\sigma^{2}\left(\underline{y_{t}}, \underline{z_{t+1}}\right), \frac{\kappa_{t}}{\gamma_{t}\left(z_{t+1}\right)}\right),
\end{aligned}
$$

where $C_{B S}$ is the (one-period) Black-Scholes formula with a volatility equal to $\sigma^{2}\left(\underline{y_{t}}, \underline{z_{t+1}}\right)$, moneyness strike equal to $\kappa_{t} / \gamma_{t}\left(z_{t+1}\right)$ and

$$
\gamma_{t}\left(z_{t+1}\right)=\exp \left[\mu\left(\underline{y_{t}}, \underline{z_{t+1}}\right)-r_{t+1}^{f}+\alpha_{t} \sigma^{2}\left(\underline{y_{t}}, \underline{z_{t+1}}\right)+\frac{\sigma^{2}\left(\underline{y_{t}}, \underline{z_{t+1}}\right)}{2}\right] \text {. }
$$

This general result can be specified for the well-known case where the latent process $z_{t}$ is an homogeneous J-states Markov chain [Markovian Switching Regimes; see Hamilton (1989)]. In this context, equation (8.3) becomes :

$$
f\left(y_{t+1}, z_{t+1} \mid \underline{y_{t}}, \underline{z_{t}}\right)=f\left(y_{t+1} \mid \underline{y_{t}}, z_{t+1}\right) \times P\left[z_{t+1} \mid z_{t}\right],
$$

and the transition probability $P\left[e_{j} \mid e_{i}\right]$ from state $e_{i}$ to state $e_{j}$ is denoted by $\pi_{i j}$.

The conditional Laplace transform of $\left(y_{t+1}, z_{t+1} \mid \underline{y_{t}}, z_{t}=e_{i}\right)$ has the form :

$$
\begin{aligned}
\varphi_{t}(u, v) & =E\left[\exp \left(v^{\prime} z_{t+1}+u \mu\left(\underline{y_{t}}, z_{t+1}\right)+\frac{1}{2} u^{2} \sigma^{2}\left(\underline{y_{t}}, z_{t+1}\right)\right) \mid \underline{y_{t}}, z_{t}=e_{i}\right] \\
& =\sum_{j=1}^{J} \pi_{i j} \exp \left[v^{\prime} e_{j}+u \mu\left(\underline{y_{t}}, e_{j}\right)+\frac{1}{2} u^{2} \sigma^{2}\left(\underline{y_{t}}, e_{j}\right)\right],
\end{aligned}
$$

and, consequently, the conditional risk-neutral Laplace transform becomes :

$$
\varphi^{Q_{t}}(u, v)=\sum_{j=1}^{J} \nu_{i j, t} \exp \left[v^{\prime} e_{j}+u\left[\mu\left(\underline{y_{t}}, e_{j}\right)+\alpha_{t} \sigma^{2}\left(\underline{y_{t}}, e_{j}\right)\right]+\frac{1}{2} u^{2} \sigma^{2}\left(\underline{y_{t}}, e_{j}\right)\right]
$$

where

$$
\nu_{i j, t}=\frac{\pi_{i j} \exp \left(\delta_{t}^{\prime} e_{j}+\alpha_{t} \mu\left(\underline{y_{t}}, e_{j}\right)+\frac{1}{2} \alpha_{t}^{2} \sigma^{2}\left(\underline{y_{t}}, e_{j}\right)\right)}{\sum_{j=1}^{J} \pi_{i j} \exp \left(\delta_{t}^{\prime} e_{j}+\alpha_{t} \mu\left(\underline{y_{t}}, e_{j}\right)+\frac{1}{2} \alpha_{t}^{2} \sigma^{2}\left(\underline{y_{t}}, e_{j}\right)\right)} .
$$

\footnotetext{
${ }^{12}$ In the literature we observe different ways to use Switching Regime methodology for option pricing : a) Campbell and $\mathrm{Li}$ (2002) specify directly the risk-neutral density of the underlying asset missing a precise analysis of options risk premia; b) Billio and Pelizzon (2000), Bollen (1998), Chourdakis and Tzavalis (2000) consider the empirically rejected assumption of idiosyncratic nature for the risk introduced by the switching of regimes $\left[\delta_{t}=0\right.$ in our case]; c) Duan, Popova and Ritchken (2002) propose a particular switching regime model where both sources of risk (the $\log$ return and the changes of regimes) are priced but with constant risk correction coefficients $\left[\alpha_{t}=\alpha\right.$ and $\delta_{t}=\delta$ in our modelisation]; d) Garcia and Renault (1998) and Garcia, Luger and Renault (2001, 2003) follow a recursive utility-based approach [Epstein-Zin (1989)], where the latent variable affects the fundamentals of the economy.
} 
So, in the risk-neutral world, the process $\left(y_{t+1}, z_{t+1}\right)$ remains a Markov-Switching process with time-varying transition probabilities and different conditional means for the normal distributions.

The specification of the pricing formula (8.6) takes the following form :

$$
C_{t}=\sum_{j=1}^{J} \nu_{i j, t} \gamma_{t}\left(e_{j}\right) C_{B S}\left(\sigma^{2}\left(e_{j}\right), \frac{\kappa_{t}}{\gamma_{t}\left(e_{j}\right)}\right),
$$

where

$$
\gamma_{t}\left(e_{j}\right)=\exp \left[\mu\left(\underline{y_{t}}, e_{j}\right)-r_{t+1}^{f}+\alpha_{t} \sigma^{2}\left(\underline{y_{t}}, e_{j}\right)+\frac{\sigma^{2}\left(\underline{y_{t}}, e_{j}\right)}{2}\right] .
$$

\subsection{Log-linear pricing with mixture component}

A general approach to the discrete-time option pricing problem, using the unifying framework provided by the Stochastic Discount Factor method [see, among others, Hansen and Richard (1987) and Cochrane (2001)], is presented by Garcia, Ghysels and Renault (2002). In this paper they show, using the SDF formulation and the Cameron-Martin-Girsanov theorem, that when the bivariate process $\left[\ln \left(M_{t, t+1}\right), \ln \left(S_{t+1} / S_{t}\right)\right]$ is conditionally normal given $I_{t}$ (Conditional Lognormality Assumption), the pricing formula derived from this class of models (named Log-linear pricing models) for a payoff $h\left(S_{t+1}\right)$ is given by :

$$
\begin{aligned}
p_{t}(h, 1) & =E_{t}\left[M_{t, t+1} h\left(S_{t+1}\right)\right] \\
& =E_{t}\left[M_{t, t+1}\right] E_{t}\left\{h\left[S_{t+1} \exp \left[\operatorname{Cov}_{t}\left(\log \left(M_{t, t+1}\right), \log \left(S_{t+1} / S_{t}\right)\right)\right]\right]\right\} .
\end{aligned}
$$

This result is generalized, in the same paper, by the introduction of a mixture component in the conditioning set which leads to the formulation of a Conditional Lognormality with mixture Assumption : if there exists a latent (mixing) variable $z_{t+1}$ such that the bivariate process $\left[\ln \left(M_{t, t+1}\right), \ln \left(S_{t+1} / S_{t}\right)\right]$ is conditionally normal given $I_{t}$ and $z_{t+1}$, the one-period pricing formula for the Log-linear model takes the following form :

$$
\begin{aligned}
p_{t}(h, 1)= & E_{t}\left[M_{t, t+1} h\left(S_{t+1}\right)\right] \\
= & E_{t}\left\{E_{t}\left[M_{t, t+1} h\left(S_{t+1}\right) \mid z_{t+1}\right]\right\} \\
= & E_{t}\left\{E_{t}\left[M_{t, t+1} \mid z_{t+1}\right]\right. \\
& \quad \times E_{t}\left[h\left(S_{t+1} \exp \left(\operatorname{Cov}_{t}\left[\left(\ln \left(M_{t, t+1}\right), \ln \left(S_{t+1} / S_{t}\right) \mid z_{t+1}\right]\right)\right) \mid z_{t+1}\right]\right\} .
\end{aligned}
$$

Now, if we consider our mixed-normal framework, characterized by a mixing latent variable $z_{t+1}$ and described by a General Switching Regime model, with $M_{t, t+1}=\exp \left(\alpha_{t} y_{t+1}+\delta_{t}^{\prime} z_{t+1}+\beta_{t}\right)$, $S_{t}=1, y_{t+1}=\ln \left(S_{t+1}\right), h\left(S_{t+1}\right)=g\left(y_{t+1}\right)=\left(\exp y_{t+1}-\kappa\right)^{+}$we observe that the Conditional Lognormality with mixture Assumption is satisfied and, consequently, our mixed-normal pricing formula (8.6) can also be derived from (8.12). Moreover, given that the i.i.d. mixture case characterizing Section 4 is a particular case of the General Switching Regimes model presented here $\left[P\left(z_{t+1}=j\right)=p_{j}, j \in\{1, \ldots, J\}\right.$, and $\left.M_{t, t+1}=\exp \left(\alpha_{t} y_{t+1}+\beta_{t}\right)\right]$, we have that also the pricing formula (4.4) can be specified from relation (8.12). 


\subsection{Pricing path dependent derivatives}

The pricing procedures presented in the previous sections can be generalized to the case of a derivative providing a general payoff of the form $g\left(y_{t+1}, \ldots, y_{t+H}\right)$ at date $t+H$. Neither the joint historical distribution of $\left(y_{t+1}, \ldots, y_{t+H}\right)$ nor the risk-neutral joint distribution (conditional to the information at time $t$ ) is in general a mixture of normal distributions. However, it is easy to simulate a path $y_{t+1}^{s}, \ldots, y_{t+H}^{s}$, function of present and past values of the associated latent variable, in the risk-neutral distribution.

More precisely, the procedure, from the investor point of view, is the following :

- given the observations $\left(y_{1}, \ldots, y_{t}\right):=\underline{y_{t}}$ and $\left(z_{1}, \ldots, z_{t}\right):=\underline{z_{t}}$, and given the values of $\alpha_{t}$ and $\delta_{t}, z_{t+1}^{s}$ is simulated from $\nu\left(z_{t+1} \mid \underline{y_{t}}, \underline{z_{t}}\right)$;

- then, $y_{t+1}^{s}$ is simulated from the one-period conditional risk-neutral distribution

$$
f^{Q}\left(y_{t+1} \mid \underline{y_{t}}, z_{t+1}^{s}, \underline{z_{t}}\right)=n\left(\mu\left(\underline{y_{t}}, z_{t+1}^{s}, \underline{z_{t}}\right)+\alpha_{t} \sigma^{2}\left(\underline{y_{t}}, z_{t+1}^{s}, \underline{z_{t}}\right), \sigma^{2}\left(\underline{y_{t}}, z_{t+1}^{s}, \underline{z_{t}}\right)\right),
$$

which is, conditionally to $z_{t+1}^{s}$, a normal distribution depending on $\left(\underline{y_{t}}, \underline{z_{t}}\right)$;

- $z_{t+2}^{s}$ is simulated from $\nu\left(z_{t+2} \mid y_{t+1}^{s}, \underline{y_{t}}, z_{t+1}^{s} \underline{z}_{t}\right)$, given the values of $\alpha_{t+1}, \delta_{t+1}$, and $y_{t+2}^{s}$ is consequently simulated from the following risk-neutral gaussian distribution

$$
\begin{gathered}
f^{Q}\left(y_{t+2} \mid y_{t+1}^{s}, \underline{y_{t}}, z_{t+2}^{s}, z_{t+1}^{s}, \underline{z_{t}}\right)=n\left(\mu\left(y_{t+1}^{s}, \underline{y_{t}}, z_{t+2}^{s}, z_{t+1}^{s}, \underline{z_{t}}\right)+\alpha_{t+1} \sigma^{2}\left(y_{t+1}^{s}, \underline{y_{t}}, z_{t+2}^{s}, z_{t+1}^{s}, \underline{z_{t}}\right),\right. \\
\left.\sigma^{2}\left(y_{t+1}^{s}, \underline{y_{t}}, z_{t+2}^{s}, z_{t+1}^{s}, \underline{z_{t}}\right)\right),
\end{gathered}
$$

and so on for the $H$ values characterizing the payoff.

Finally, using $S$ simulated paths, the price at time $t$ of the derivative, in the case of short-term risk-free rates known at $t$, is approximated by :

$$
\exp \left(-\sum_{h=1}^{H} r_{t+h}^{f}\right) \frac{1}{S} \sum_{s=1}^{S} g\left(y_{t+1}^{s}, \ldots, y_{t+H}^{s}\right) .
$$

This formula is valid for any path dependent derivative. In the simple case of a European Call option (with $S_{t}=1$ ) it becomes :

$$
\exp \left(-\sum_{h=1}^{H} r_{t+h}^{f}\right) \frac{1}{S} \sum_{s=1}^{S}\left[\exp \left(y_{t+1}^{s}+\ldots+y_{t+H}^{s}\right)-\kappa_{t}\right]^{+} .
$$

\subsection{Switching regimes and implied volatility surface}

In previous sections we have presented the General Switching Regime model for option pricing, a generalization of i.i.d. mixtures case [see section 4] where a white noise dynamics was assumed for the latent variable $z_{t+1}$. For this i.i.d. case we have verified the ability of the Mixed-Normal static model to replicate smiles and volatility skews coherent with empirical results.

Nevertheless, this model show some limit about the possibility to build implied volatility surfaces close to the observed ones. More precisely, as indicated by empirical evidence [see, among the others, Cont and da Fonseca (2002)], these surfaces are sometimes characterized by shapes 
with smiles (or volatility skews) also for large maturities, while the surface obtained by the MixedNormal static model gives for large maturities flat implied Black-Scholes volatilities [see Figure 4].

Consequently, it could be interesting to verify, by the introduction of serial dependence in the dynamics of the latent variable, if we are able to replicate these observed implied volatility surfaces.

We consider a Hidden Markov Chain (HMC) specification for the General Switching Regime model ${ }^{13}$, where the dynamics of $z_{t+1}$ is described by a two-states homogeneous Markov chain. We assume $\alpha_{t}=\alpha$ and $\delta_{t}=\delta$, and we specify a symmetric switching in $\delta\left[\delta_{1}=-\delta_{2}=d\right.$, with $\left.d \in \mathbb{R}^{+}\right]$. In this way, we obtain a nonlinear system of two equations with two unknowns for the absence of arbitrage restrictions.

We consider a mixture of two gaussian distributions with the same means $\left(\mu_{1}=\mu_{2}=0.03\right)$, with the variance of the first component fixed to $\sigma_{1}^{2}=0.03$ (low volatility regime) and that of the second component fixed to $\sigma_{2}^{2}=0.04$ (high volatility regime), and we consider the regime persistence probabilities at levels coherent with empirical evidence on stock markets : $\pi_{11}=0.99$ and $\pi_{22}=0.95$. The implied volatility surface obtained by Monte Carlo simulations is presented in Figure 8 (the time to maturity, measured in years, changes from 0.25 to 1.5 ). We observe that the volatility skew (typical of stock markets) characterizes not only the small maturities but also the large ones, giving, in general, a surface coherent with the observations.

Therefore, an HMC specification provides a model able to replicate interesting phenomena like the shapes of the implied volatility surfaces and, in particular, the introduction of serial dependence in the dynamics of $z_{t+1}$ seems to be the element giving to the HMC option pricing model the possibility to produce better performances (in simulation) with respect to the Mixed-Normal static specification.

\section{Concluding remarks}

This paper has developed a global discrete time option pricing methodology when the parametric SDF is exponential-affine and the geometric return of the underlying asset has a dynamics characterized by a Mixture of Conditionally Normal processes.

This methodology offers a flexible and promising framework to build prices of European and path dependent options as indicated by the possibility to derive explicit risk-neutral probability measures under various model specifications. More precisely, the Mixed-Normal framework, combined with the (exponential-affine) SDF modeling principle, is developed in static and dynamic models with parametric, semi-parametric and nonparametric specifications, and illustrated by explicit derivative pricing formulas and implied Black-Scholes volatilities. The numerical analysis has confirmed the ability of our general option pricing approach to replicate smiles, volatility skews and implied volatility surfaces coherent with empirical results. The purpose of future research will be to test the models presented in the paper from observations of spot and option prices.

\footnotetext{
${ }^{13}$ Given the observable variable $y_{t+1}$ and the regime indicator (hidden) variable $z_{t+1}$ taking values $e_{j}=$ $[0, \ldots, 1, \ldots, 0]^{\prime}, j \in\{1, \ldots, J\}$, the stochastic process $\left(y_{t+1}, z_{t+1}\right)$ defines a Hidden Markov Chain (HMC) model if its conditional distribution satisfies the following property :

$$
\begin{aligned}
f\left(y_{t+1}, z_{t+1} \mid \underline{y_{t}}, \underline{z_{t}}\right) & =f\left(y_{t+1} \mid \underline{y_{t}}, \underline{z_{t+1}}\right) f\left(z_{t+1} \mid \underline{y_{t}}, \underline{z_{t}}\right) \\
& =f\left(y_{t+1} \mid z_{t+1}\right) \times f\left(z_{t+1} \mid z_{t}\right),
\end{aligned}
$$

that is, if we assume that $z_{t+1}$ is an homogeneous J-states Markov chain, and if we assume that, for $y_{t+1}$, the relevant information contained in $\left(\underline{y_{t}}, \underline{z_{t+1}}\right)$ is summarized in $z_{t+1}$.
} 


\section{Appendix 1 \\ Existence of a mixture of two Normal distributions reaching any pair of skewness-kurtosis in $\mathcal{D}$}

Let us first recall the general relation between skewness and kurtosis of a random variable with any distribution : $\tilde{\mu}_{4} \geq \tilde{\mu}_{3}^{2}+1$.

Moreover, observe that skewness and kurtosis are stable with respect to an affine transformation of the random variable. This means that, we can study the problem of spanning pairs of skewnesskurtosis in $\mathcal{D}$ for standardized mixed-normal random variables. We will consider also an affine transformation of these variables in order to show the ability of a mixture of Normal distributions to span any set of $\left(\mu, \sigma^{2}, \tilde{\mu}_{3}, \tilde{\mu}_{4}\right)$.

\section{a) Spanning the boundary of $\mathcal{D}$}

The boundary of the maximal set $\mathcal{D}$ can be attained by the following special type of mixture (with $p \in] 0,1[):$

$$
p \mathcal{N}\left(\sqrt{\frac{1-p}{p}}, 0\right)+(1-p) \mathcal{N}\left(-\sqrt{\frac{p}{1-p}}, 0\right)
$$

indeed, this discrete (two-values) random variable has the following first four moments :

$$
\begin{aligned}
\mu_{o} & =0 \\
\sigma_{o}^{2} & =1 \\
\tilde{\mu}_{3}^{o} & =\frac{1-2 p}{\sqrt{p(1-p)}}, \text { describing } \mathbb{R}, \\
\tilde{\mu}_{4}^{o} & =\frac{1}{p(1-p)}-3=\left(\tilde{\mu}_{3}^{o}\right)^{2}+1 .
\end{aligned}
$$

\section{b) Spanning the interior of $\mathcal{D}$}

Case 1 : Let us introduce in the mixture presented above a variance term, denoted $\bar{\sigma}^{2}$, common to the two Normal components. Applying the general formulas of section 4.1.2, we get the following first four moments :

$$
\begin{aligned}
\mu & =0 \\
\sigma^{2} & =\sigma_{o}^{2}+\bar{\sigma}^{2}=1+\bar{\sigma}^{2} \\
\tilde{\mu}_{3} & =\frac{\tilde{\mu}_{3}^{o}}{\left(1+\bar{\sigma}^{2}\right)^{\frac{3}{2}}} \\
\tilde{\mu}_{4} & =\frac{\tilde{\mu}_{4}^{o}+3 \bar{\sigma}^{4}+6 \bar{\sigma}^{2}}{\left(1+\bar{\sigma}^{2}\right)^{2}} \\
& =\frac{\left(\tilde{\mu}_{3}^{o}\right)^{2}+1+3 \bar{\sigma}^{4}+6 \bar{\sigma}^{2}}{\left(1+\bar{\sigma}^{2}\right)^{2}} \\
& =\left(1+\bar{\sigma}^{2}\right) \tilde{\mu}_{3}^{2}+3-\frac{2}{\left(1+\bar{\sigma}^{2}\right)^{2}} .
\end{aligned}
$$

Now, we can consider two cases. 
If $\tilde{\mu}_{3} \neq 0$, or $\tilde{\mu}_{3}=0$ with $\tilde{\mu}_{4}<3$, it is possible to reach the pair $\left(\tilde{\mu}_{3}, \tilde{\mu}_{4}\right)$ in the interior of $\mathcal{D}$ [denoted $\operatorname{Int}(\mathcal{D})]$ thanks to the choice of $\bar{\sigma}^{2}$. More precisely, denoting $x=1+\bar{\sigma}^{2}$, we look for the (unique) root $a>1$, of the following equation in $x$ :

$$
\begin{gathered}
\tilde{\mu}_{4}=x\left(\tilde{\mu}_{3}\right)^{2}+3-\frac{2}{x^{2}} \\
\Longleftrightarrow \quad\left(\tilde{\mu}_{3}\right)^{2} x^{3}+\left(3-\tilde{\mu}_{4}\right) x^{2}-2=0 .
\end{gathered}
$$

Given that the fixed pair $\left(\tilde{\mu}_{3}, \tilde{\mu}_{4}\right) \in \operatorname{Int}(\mathcal{D})$, the polynomial $p(x)=\left(\tilde{\mu}_{3}\right)^{2} x^{3}+\left(3-\tilde{\mu}_{4}\right) x^{2}-2$ takes the negative value $\left(\tilde{\mu}_{3}\right)^{2}+1-\tilde{\mu}_{4}$ for $x=1$ and converges to $+\infty$ when $x \rightarrow+\infty$, which proves the existence of the desired root $a$ (it is easy to verify the uniqueness of $a$ ). Now it just remains to look for the value of $p$ satisfying the relation :

$$
\frac{1-2 p}{\sqrt{p(1-p)}}=\tilde{\mu}_{3}^{o}=a^{\frac{3}{2}} \tilde{\mu}_{3}
$$

this leads to :

$$
p=\frac{1}{2}-\frac{\tilde{\mu}_{3}^{o}}{2 \sqrt{\left(\tilde{\mu}_{3}^{o}\right)^{2}+4}} .
$$

So, here is the corresponding mixture having skewness $\tilde{\mu}_{3}$ and kurtosis $\tilde{\mu}_{4}$ :

$$
p \mathcal{N}\left(\sqrt{\frac{1-p}{p}}, a-1\right)+(1-p) \mathcal{N}\left(\sqrt{-\frac{p}{(1-p)}}, a-1\right)
$$

To complete the analysis of this case, let us mention that we can apply an affine transformation to obtain also a desired pair of mean-variance. And this gives the following mixture :

$$
p \mathcal{N}\left(\mu+\sigma \sqrt{\frac{1-p}{a p}}, \frac{\sigma^{2}(a-1)}{a}\right)+(1-p) \mathcal{N}\left(\mu-\sigma \sqrt{\frac{p}{a(1-p)}}, \frac{\sigma^{2}(a-1)}{a}\right) .
$$

Case 2 : In the case where $\tilde{\mu}_{3}=0$ with $\tilde{\mu}_{4} \geq 3$, the polynomial above has no more real roots. However, it is straightforward to verify that the following mixture has its first four moments equal to $\mu, \sigma^{2}, \tilde{\mu}_{3}=0, \tilde{\mu}_{4} \geq 3$ :

$$
p \mathcal{N}\left(\mu, \frac{\sigma^{2}}{2 p}\right)+(1-p) \mathcal{N}\left(\mu, \frac{\sigma^{2}}{2(1-p)}\right)
$$

with $p=\frac{1}{2} \pm \frac{1}{2} \sqrt{1-\frac{3}{\tilde{\mu}_{4}}}$. 


\section{Appendix 2}

\section{Existence and uniqueness of alpha}

If we consider the absence of arbitrage condition $\varphi(\alpha+1)=\exp (r) \varphi(\alpha)$ for the particular case of $r=0$, we find in the mixed-normal framework the following relation :

$$
H(\alpha) \equiv \sum_{j=1}^{J} p_{j} \exp \left(\alpha \mu_{j}+\sigma_{j}^{2} \frac{\alpha^{2}}{2}\right)\left[\exp \left(\mu_{j}+\sigma_{j}^{2} \alpha+\frac{\sigma_{j}^{2}}{2}\right)-1\right]=0,
$$

and we immediately see the existence of a solution since :

$$
\lim _{\alpha \rightarrow+\infty} H(\alpha)=+\infty, \quad \lim _{\alpha \rightarrow-\infty} H(\alpha)=-\infty
$$

Now, we have to verify that the solution is unique. In order to obtain this result we write the first derivative of function $H(\alpha)$ :

$$
\begin{aligned}
H^{\prime}(\alpha)= & \sum_{j=1}^{J} p_{j} \exp \left(\alpha \mu_{j}+\sigma_{j}^{2} \frac{\alpha^{2}}{2}\right) \\
& \left\{\left(\mu_{j}+\sigma_{j}^{2} \alpha\right)\left[\exp \left(\mu_{j}+\sigma_{j}^{2} \alpha+\frac{\sigma_{j}^{2}}{2}\right)-1\right]+\sigma_{j}^{2} \exp \left(\mu_{j}+\sigma_{j}^{2} \alpha+\frac{\sigma_{j}^{2}}{2}\right)\right\} .
\end{aligned}
$$

If we consider the function :

$$
h(x)=x\left[\exp \left(x+\frac{a}{2}\right)-1\right]+a \exp \left(x+\frac{a}{2}\right),
$$

with $a>0$, it is easy to verify that $h$ is strictly positive for every value of $x$; consequently, taking $x:=\mu_{j}+\sigma_{j}^{2} \alpha$ and $a:=\sigma_{j}^{2}$, we see that $H^{\prime}(\alpha)$ is strictly positive for every value of $\alpha$ and $H(\alpha)$ is strictly increasing: therefore, the value of $\alpha$ such that $\varphi(\alpha+1)=\varphi(\alpha)$ is unique.

Now, let us consider the general case of $r>0$. The relation $H(\alpha)$ takes the following form :

$$
H(\alpha)=\sum_{j=1}^{J} p_{j} \exp \left(\alpha \mu_{j}+\sigma_{j}^{2} \frac{\alpha^{2}}{2}\right)\left[\exp \left(\mu_{j}+\sigma_{j}^{2} \alpha+\frac{\sigma_{j}^{2}}{2}\right)-\exp r\right]=0
$$

we can rewrite relation (A.4) in this way :

$$
\exp (r) \sum_{j=0}^{J} p_{j} \exp \left(\alpha \mu_{j}+\sigma_{j}^{2} \frac{\alpha^{2}}{2}\right)\left[\exp \left(\mu_{j}-r+\sigma_{j}^{2} \alpha+\frac{\sigma_{j}^{2}}{2}\right)-1\right]=0
$$

which is equivalent to

$$
\exp (r+\alpha r) \sum_{j=1}^{J} p_{j} \exp \left(\alpha \mu_{j}^{\prime}+\sigma_{j}^{2} \frac{\alpha^{2}}{2}\right)\left[\exp \left(\mu_{j}^{\prime}+\sigma_{j}^{2} \alpha+\frac{\sigma_{j}^{2}}{2}\right)-1\right]=0
$$

with $\mu_{j}^{\prime}=\mu_{j}-r$, and we obtain the same relation as in the case of $r=0$ multiplied by the positive quantity $\exp (r+\alpha r)$. This result leads us to the general conclusion that the value $\alpha$ is unique for every value of $r \geq 0$ and $\left(\mu_{j}, \sigma_{j}^{2}, p_{j}\right), j=1, \ldots, J$. 


\section{Appendix 3}

\section{The risk-neutral distribution}

From asset pricing theory we know that the specification of the risk-neutral distribution through the SDF change of measure is given by :

$$
f^{Q}(y)=\frac{M}{E(M)} f(y)
$$

In our framework this relation takes the following form :

$$
\begin{aligned}
f^{Q}(y) & =\frac{f(y) \exp (\alpha y)}{\sum_{j=1}^{J} p_{j} \exp \left(\alpha \mu_{j}+\alpha^{2} \frac{\sigma_{j}^{2}}{2}\right)} \\
& =\frac{f(y) \exp (\alpha y)}{\varphi(\alpha)},
\end{aligned}
$$

where $f(y)$ is given by equation (4.1) and $\varphi(\alpha)$ is the Laplace transform of a mixture of normal distributions. Now, we can write equation (A.7) in the following way :

$$
\begin{aligned}
f^{Q}(y) & =\frac{\sum_{j=1}^{J} p_{j} \frac{1}{\sigma_{j} \sqrt{2 \pi}} \exp \left(-\frac{\left(y-\mu_{j}\right)^{2}}{2 \sigma_{j}^{2}}+\alpha y\right)}{\varphi(\alpha)} \\
& =\frac{\sum_{j=1}^{J} p_{j} \frac{1}{\sigma_{j} \sqrt{2 \pi}} \exp \left[-\frac{\left(y-\mu_{j}-\alpha \sigma_{j}^{2}\right)^{2}}{2 \sigma_{j}^{2}}+\alpha \mu_{j}+\frac{\alpha^{2}}{2} \sigma_{j}^{2}\right]}{\varphi(\alpha)} \\
& =\sum_{j=1}^{J}\left[\frac{p_{j} \exp \left(\alpha \mu_{j}+\frac{\alpha^{2}}{2} \sigma_{j}^{2}\right)}{\sum_{j=1}^{J} p_{j} \exp \left(\alpha \mu_{j}+\alpha^{2} \frac{\sigma_{j}^{2}}{2}\right)}\right] \frac{\exp \left[-\frac{\left(y-\mu_{j}-\alpha \sigma_{j}^{2}\right)^{2}}{2 \sigma_{j}^{2}}\right]}{\sigma_{j} \sqrt{2 \pi}} \\
& =\sum_{j=1}^{J} \nu_{j} f_{j}\left(y ; \mu_{j}+\alpha \sigma_{j}^{2}, \sigma_{j}^{2}\right) .
\end{aligned}
$$

The risk-neutral distribution is still a mixture of normal distributions with new means $\mu_{j}+\alpha \sigma_{j}^{2}$ and the same variances $\sigma_{j}^{2}$, but characterized by a new mixing distribution (risk-adjusted mixing distribution) $\nu_{j}, j=1, \ldots, J$. 


\section{Appendix 4}

\section{The option pricing formula}

We have seen in Proposition 3 (and in Appendix 3) that the unique risk-neutral distribution $Q$ associated to the historical distribution (4.1) is once more a mixture of gaussian distributions. This result allows to write the pricing formula as :

$$
\begin{aligned}
C_{t} & =\exp (-r) E^{Q}\left[\exp y_{t+1}-\kappa\right]^{+} \\
& =\exp (-r) \sum_{j=1}^{J} \nu_{j} E\left[\exp \left(y_{j}\right)-\kappa\right]^{+},
\end{aligned}
$$

with $y_{j} \sim \mathcal{N}\left[\mu_{j}+\alpha \sigma_{j}^{2}, \sigma_{j}^{2}\right]$ for every $t$. Now, this gaussian random variable can be decomposed in the following sum :

$$
y_{j}=z_{j}+\mu_{j}+\alpha \sigma_{j}^{2}-r+\frac{\sigma_{j}^{2}}{2}
$$

with $z_{j} \sim \mathcal{N}\left[r-\frac{\sigma_{j}^{2}}{2}, \sigma_{j}^{2}\right]$. This decomposition gives us the possibility to write the formula as an average of Black-Scholes pricing formulas :

$$
C_{t}=\exp (-r) \sum_{j=1}^{J} \nu_{j} E\left[\gamma_{j} \exp \left(z_{j}\right)-\kappa\right]^{+}
$$

with $\gamma_{j}=\exp \left(\mu_{j}+\alpha \sigma_{j}^{2}-r+\frac{\sigma_{j}^{2}}{2}\right)$, and consequently

$$
\begin{aligned}
C_{t} & =\exp (-r) \sum_{j=1}^{J} \nu_{j} \gamma_{j} E\left[\exp \left(z_{j}\right)-\frac{\kappa}{\gamma_{j}}\right]^{+} \\
& =\sum_{j=1}^{J} \nu_{j} \gamma_{j} C_{B S}\left(\sigma_{j}^{2}, \frac{\kappa}{\gamma_{j}}\right)
\end{aligned}
$$

with $C_{B S}\left(\sigma^{2}, \kappa\right)$ the one-period Black-Scholes formula

$$
C_{B S}\left(\sigma^{2}, \kappa\right)=\left[\Phi\left(-\frac{\ln \left(\kappa e^{-r}\right)}{\sigma}+\frac{\sigma}{2}\right)-\kappa e^{-r} \Phi\left(-\frac{\ln \left(\kappa e^{-r}\right)}{\sigma}-\frac{\sigma}{2}\right)\right]
$$

and

$$
\begin{aligned}
\nu_{j} \gamma_{j} & =\frac{p_{j} \exp \left(\alpha \mu_{j}^{\prime}+\sigma_{j}^{2} \frac{\alpha^{2}}{2}\right) \exp \left(\mu_{j}^{\prime}+\alpha \sigma_{j}^{2}+\frac{\sigma_{j}^{2}}{2}\right)}{\sum_{j=1}^{J} p_{j} \exp \left(\alpha \mu_{j}^{\prime}+\sigma_{j}^{2} \frac{\alpha^{2}}{2}\right)} \\
\mu_{j}^{\prime} & :=\mu_{j}-r
\end{aligned}
$$


Moreover we have $\sum_{j=1}^{J} \nu_{j} \gamma_{j}=1$. Indeed, because of formula (A.5), we can write :

$$
\begin{aligned}
\sum_{j=1}^{J} \nu_{j} \gamma_{j}= & \frac{\sum_{j=1}^{J} p_{j} \exp \left(\alpha \mu_{j}^{\prime}+\sigma_{j}^{2} \frac{\alpha^{2}}{2}\right) \exp \left(\mu_{j}^{\prime}+\alpha \sigma_{j}^{2}+\frac{\sigma_{j}^{2}}{2}\right)}{\sum_{j=1}^{J} p_{j} \exp \left(\alpha \mu_{j}^{\prime}+\sigma_{j}^{2} \frac{\alpha^{2}}{2}\right)} \\
= & \frac{\sum_{j=1}^{J} p_{j} \exp \left(\alpha \mu_{j}^{\prime}+\sigma_{j}^{2} \frac{\alpha^{2}}{2}\right)}{\sum_{j=1}^{J} p_{j} \exp \left(\alpha \mu_{j}^{\prime}+\sigma_{j}^{2} \frac{\alpha^{2}}{2}\right)}=1 .
\end{aligned}
$$

\section{Appendix 5}

\section{Modeling extreme risks}

Proof of Proposition 5 : For a given $\lambda \in] 0,1[$, the Fourier transform of the p.d.f. (4.5), denoted $\tilde{\varphi}_{\lambda}(u)$, is given by :

$$
\begin{aligned}
\tilde{\varphi}_{\lambda}(u) & =E[\exp (i u y)] \\
& =\lambda \exp \left(i u \mu-\frac{u^{2}}{2} \frac{\sigma_{1}^{2}}{\lambda}\right)+(1-\lambda) \exp \left(i u \mu-\frac{u^{2}}{2} \frac{\sigma_{2}^{2}}{1-\lambda}\right) ;
\end{aligned}
$$

now, in the extreme risk case, the above relation becomes :

$$
\lim _{\lambda \rightarrow 0} \tilde{\varphi}_{\lambda}(u)=\exp \left(i u \mu-\frac{u^{2}}{2} \sigma_{2}^{2}\right), \quad \forall u \in \mathbb{R},
$$

which is the Fourier transform of the Gaussian random variable $\mathcal{N}\left(\mu, \sigma_{2}^{2}\right)$. Consequently, the logreturn $y$ with p.d.f. (4.5) converges in distribution to $\mathcal{N}\left(\mu, \sigma_{2}^{2}\right)$ when $\lambda \rightarrow 0$ [the proof of the other results in Proposition 4 is straightforward].

Proof of Proposition 6 : Let us recall the following relation (A.4) of Appendix 1 applied in this particular case :

$$
\begin{aligned}
& H_{\lambda}(\alpha) \equiv \lambda \exp \left(\frac{\sigma_{1}^{2}}{\lambda} \frac{\alpha^{2}}{2}\right)\left[\exp \left(\mu+\frac{\sigma_{1}^{2}}{\lambda} \alpha+\frac{\sigma_{1}^{2}}{2 \lambda}\right)-\exp (r)\right]+ \\
& (1-\lambda) \exp \left(\frac{\sigma_{2}^{2}}{1-\lambda} \frac{\alpha^{2}}{2}\right)\left[\exp \left(\mu+\frac{\sigma_{2}^{2}}{1-\lambda} \alpha+\frac{\sigma_{2}^{2}}{2(1-\lambda)}\right)-\exp (r)\right]=0 .
\end{aligned}
$$


In particular,

$$
\begin{aligned}
& H_{\lambda}\left(-\frac{1}{2}+\epsilon\right) \equiv \lambda \exp \left(\frac{\sigma_{1}^{2}}{\lambda} \frac{\left(-\frac{1}{2}+\epsilon\right)^{2}}{2}\right)\left[\exp \left(\mu+\epsilon \frac{\sigma_{1}^{2}}{\lambda}\right)-\exp (r)\right]+ \\
& (1-\lambda) \exp \left(\frac{\sigma_{2}^{2}}{1-\lambda} \frac{\left(-\frac{1}{2}+\epsilon\right)^{2}}{2}\right)\left[\exp \left(\mu+\epsilon \frac{\sigma_{2}^{2}}{1-\lambda}\right)-\exp (r)\right] .
\end{aligned}
$$

If $\epsilon>0: H_{\lambda}\left(-\frac{1}{2}+\epsilon\right) \rightarrow+\infty$ when $\lambda \rightarrow 0$.

If $\epsilon<0: H_{\lambda}\left(-\frac{1}{2}+\epsilon\right) \rightarrow-\infty$ when $\lambda \rightarrow 0$.

As $H_{\lambda}$ is an increasing function, we can deduce that :

$\forall \epsilon>0$, for $\lambda$ sufficiently small, the solution $\alpha$ of the equation lies in the interval $-\frac{1}{2} \pm \epsilon$.

So $\alpha \rightarrow-\frac{1}{2}$ when $\lambda \rightarrow 0$.

Proof of Proposition 8 : We have written the price of a European Call option as a direct application of formula (4.4) :

$$
C_{t}=\nu_{1} \gamma_{1} C_{B S}\left(\frac{\sigma_{1}^{2}}{\lambda}, \frac{\kappa}{\gamma_{1}}\right)+\nu_{2} \gamma_{2} C_{B S}\left(\frac{\sigma_{2}^{2}}{1-\lambda}, \frac{\kappa}{\gamma_{2}}\right)
$$

with

$$
\begin{aligned}
\nu_{1} \gamma_{1} & =\frac{\lambda \exp \left(\frac{\sigma_{1}^{2}}{2 \lambda}(\alpha+1)^{2}\right)}{\lambda \exp \left(\frac{\sigma_{1}^{2}}{2 \lambda}(\alpha+1)^{2}\right)+(1-\lambda) \exp \left(\frac{\sigma_{2}^{2}}{2(1-\lambda)}(\alpha+1)^{2}\right)} \\
& \rightarrow \quad 1 \quad \text { when } \quad \lambda \rightarrow 0 .
\end{aligned}
$$

The second term $\nu_{2} \gamma_{2} C_{B S}\left(\frac{\sigma_{2}^{2}}{1-\lambda}, \frac{\kappa}{\gamma_{2}}\right)$ tends to 0 because $\nu_{2} \gamma_{2}$ tends to 0 and $C_{B S}\left(\frac{\sigma_{2}^{2}}{1-\lambda}, \frac{\kappa}{\gamma_{2}}\right)$ is bounded. Now for the first term : $\gamma_{1} \rightarrow 1$ because $\nu_{1} \rightarrow 1$ (see Proposition 7) and $\nu_{1} \gamma_{1} \rightarrow 1$. So, $C_{B S}\left(\frac{\sigma_{1}^{2}}{\lambda}, \frac{\kappa}{\gamma_{1}}\right) \rightarrow 1$. Therefore $C_{t} \rightarrow 1$.

\section{Appendix 6}

\section{The jump-diffusion model}

Proof of Proposition 9.a : Using the absence of arbitrage condition $\varphi(\alpha+1)=\exp (r) \varphi(\alpha)$ we successively find :

$$
\begin{aligned}
& \sum_{j=0}^{+\infty} e^{-\lambda} \frac{\lambda^{j}}{j !} \exp \left((\alpha+1)\left(\mu+j \mu_{p}\right)+\left(\sigma^{2}+j \sigma_{p}^{2}\right) \frac{(\alpha+1)^{2}}{2}\right) \\
& =\exp (r) \sum_{j=0}^{+\infty} e^{-\lambda} \frac{\lambda^{j}}{j !} \exp \left(\alpha\left(\mu+j \mu_{p}\right)+\left(\sigma^{2}+j \sigma_{p}^{2}\right) \frac{\alpha^{2}}{2}\right) \\
& \exp \left(\mu+\left(\alpha+\frac{1}{2}\right) \sigma^{2}\right) \sum_{j=0}^{+\infty} \frac{\left[\lambda \exp \left((\alpha+1) \mu_{p}+\sigma_{p}^{2} \frac{(\alpha+1)^{2}}{2}\right)\right]^{j}}{j !} \\
& =\exp (r) \sum_{j=0}^{+\infty} \frac{\left[\lambda \exp \left(\alpha \mu_{p}+\sigma_{p}^{2} \frac{\alpha^{2}}{2}\right)\right]^{j}}{j !}
\end{aligned}
$$


then

$$
\begin{aligned}
& \exp \left(\mu+\left(\alpha+\frac{1}{2}\right) \sigma^{2}\right) \exp \left[\lambda \exp \left((\alpha+1) \mu_{p}+\sigma_{p}^{2} \frac{(\alpha+1)^{2}}{2}\right)\right] \\
& =\exp (r) \exp \left[\lambda \exp \left(\alpha \mu_{p}+\sigma_{p}^{2} \frac{\alpha^{2}}{2}\right)\right]
\end{aligned}
$$

and we finally get

$$
\mu-r+\left(\alpha+\frac{1}{2}\right) \sigma^{2}+\lambda \exp \left(\alpha \mu_{p}+\sigma_{p}^{2} \frac{\alpha^{2}}{2}\right)\left[\exp \left(\mu_{p}+\sigma_{p}^{2}\left(\alpha+\frac{1}{2}\right)\right)-1\right]=0
$$

Proof of Proposition 9.b : Applying formula (4.3) we get the risk-neutral distribution with weights given by :

$$
\begin{aligned}
\nu_{j} & =\frac{\exp (-\lambda) \frac{\lambda^{j}}{j !} \exp \left(\alpha\left(\mu+j \mu_{p}\right)+\left(\sigma^{2}+j \sigma_{p}^{2}\right) \frac{\alpha^{2}}{2}\right)}{\sum_{j=0}^{+\infty} \exp (-\lambda) \frac{\lambda^{j}}{j !} \exp \left(\alpha\left(\mu+j \mu_{p}\right)+\left(\sigma^{2}+j \sigma_{p}^{2}\right) \frac{\alpha^{2}}{2}\right)} \\
& =\frac{\frac{\left(\lambda \exp \left(\alpha \mu_{p}+\sigma_{p}^{2} \frac{\alpha^{2}}{2}\right)\right)^{j}}{j !}}{\sum_{j=0}^{+\infty} \frac{\left(\lambda \exp \left(\alpha \mu_{p}+\sigma_{p}^{2} \frac{\alpha^{2}}{2}\right)\right)^{j}}{j !}} \\
& =\exp \left(-\lambda^{\prime}\right) \frac{\lambda^{\prime j}}{j !},
\end{aligned}
$$

with $\lambda^{\prime}=\lambda \exp \left(\alpha \mu_{p}+\sigma_{p}^{2} \frac{\alpha^{2}}{2}\right)$.

Proof of Proposition 9.c : We now apply formula (4.4) to get the price of the European Call option which is an average of the Black-Scholes formulas with weights :

$$
\beta_{j}=\nu_{j} \gamma_{j}
$$

where $\quad \gamma_{j}=\exp \left(\mu+j \mu_{p}+\alpha\left(\sigma^{2}+j \sigma_{p}^{2}\right)-r+\frac{\sigma^{2}+j \sigma_{p}^{2}}{2}\right)$

Using relation (A.8) above we get

$$
\begin{aligned}
\gamma_{j}= & \exp \left[-\lambda \exp \left((\alpha+1) \mu_{p}+\sigma_{p}^{2} \frac{(\alpha+1)^{2}}{2}\right)+\lambda \exp \left(\alpha \mu_{p}+\sigma_{p}^{2} \frac{\alpha^{2}}{2}\right)\right] \\
& \times\left[\exp \left(\mu_{p}+\alpha \sigma_{p}^{2}+\frac{\sigma_{p}^{2}}{2}\right)\right]^{j}
\end{aligned}
$$

So,

$$
\beta_{j}=\exp \left(-\lambda \exp \left((\alpha+1) \mu_{p}+\sigma_{p}^{2} \frac{(\alpha+1)^{2}}{2}\right)\right) \frac{\left[\lambda \exp \left((\alpha+1) \mu_{p}+\sigma_{p}^{2} \frac{(\alpha+1)^{2}}{2}\right)\right]^{j}}{j !}
$$




\section{Appendix 7 \\ The MN-GARCH process of first type}

If we consider the case of a mixture of $J$ components, the model presented by HMP [Hass, Mittnick and Paolella (2002)] takes the following form :

$$
\varepsilon_{t+1} \mid \underline{\varepsilon_{t}} \sim \mathcal{M N}\left(J, p_{j}, \mu_{j}, \sigma_{j t+1}^{2}\right)
$$

where $\mu_{J}=-\sum_{i=1}^{J-1}\left(p_{i} / p_{J}\right) \mu_{i}$ and where the $J \times 1$ vector of variances, denoted by $\sigma_{t+1}^{2}$, evolves according to :

$$
\sigma_{t+1}^{2}=\omega+\sum_{i=0}^{q-1} \mathcal{B}_{i+1} \varepsilon_{t-i}^{2}+\sum_{j=0}^{p-1} \mathcal{C}_{j+1} \sigma_{t-j}^{2}
$$

with $\sigma_{t+1}^{2}=\left[\sigma_{1 t+1}^{2}, \ldots, \sigma_{J t+1}^{2}\right]^{\prime}, \omega=\left[\omega_{1}, \ldots, \omega_{J}\right]^{\prime}, \mathcal{B}_{i}=\left[b_{i 1}, \ldots, b_{i J}\right]^{\prime}, i=1, \ldots, q$, and $\mathcal{C}_{j}, j=$ $1, \ldots, p$, are $J \times J$ matrices with typical element $c_{j, m n}$. Non-negativity conditions on the parameters are assumed.

The special case of $J=2$ and $p=q=1$ can be represented in the following way :

$$
\left[\begin{array}{c}
\sigma_{1 t+1}^{2} \\
\sigma_{2 t+1}^{2}
\end{array}\right]=\left[\begin{array}{l}
\omega_{1} \\
\omega_{2}
\end{array}\right]+\left[\begin{array}{l}
b_{1} \\
b_{2}
\end{array}\right] \varepsilon_{t}^{2}+\left[\begin{array}{ll}
c_{11} & c_{12} \\
& \\
c_{21} & c_{22}
\end{array}\right]\left[\begin{array}{c}
\sigma_{1 t}^{2} \\
\sigma_{2 t}^{2}
\end{array}\right],
$$

which is our specification if we impose on the parameters the constrains $c_{12}=c_{21}=0$.

In their paper, HMP note that the Diagonal MN-GARCH model, with $\mathcal{C}_{j}, j=1, \ldots, p$, a diagonal matrix, fits well the data employing various model-selection criteria [see HMP (2002) for details].

If we impose the constraint $c_{11}=c_{22}, \varepsilon_{t+1}$ has a GARCH structure; indeed, if we have :

$$
E\left(\varepsilon_{t+1} \mid \underline{\varepsilon_{t}}\right)=\lambda a(1-\lambda)-\lambda a(1-\lambda)=0
$$

and

$$
\begin{aligned}
\sigma_{t+1}^{2}=E\left(\varepsilon_{t+1}^{2} \mid \underline{\varepsilon_{t}}\right)= & \lambda\left[a^{2}(1-\lambda)^{2}+\sigma_{1 t+1}^{2}\right]+(1-\lambda)\left[a^{2} \lambda^{2}+\sigma_{2 t+1}^{2}\right] \\
= & a^{2} \lambda(1-\lambda)+\lambda \sigma_{1 t+1}^{2}+(1-\lambda) \sigma_{2 t+1}^{2} \\
= & \lambda \omega_{1}+(1-\lambda) \omega_{2}+a^{2} \lambda(1-\lambda)+\left[\lambda b_{1}+(1-\lambda) b_{2}\right] \varepsilon_{t}^{2} \\
& +c_{1} \lambda \sigma_{1 t}^{2}+c_{2}(1-\lambda) \sigma_{2 t}^{2} ;
\end{aligned}
$$


now, if we impose $c_{1}=c_{2}=c$ we can write :

$$
\begin{aligned}
\sigma_{t+1}^{2}=E\left(\varepsilon_{t+1}^{2} \mid \underline{\varepsilon_{t}}\right)= & (1-c) a^{2} \lambda(1-\lambda)+\lambda \omega_{1}+(1-\lambda) \omega_{2} \\
& +\left[\lambda b_{1}+(1-\lambda) b_{2}\right] \varepsilon_{t}^{2}+c \sigma_{t}^{2}
\end{aligned}
$$

with $\xi:=(1-c) a^{2} \lambda(1-\lambda)+\lambda \omega_{1}+(1-\lambda) \omega_{2}$ the (positive) constant term of the relation.

\section{Appendix 8}

\section{The Switching Regimes Option Pricing Model}

Proof of Proposition 16 : The Laplace transform $\varphi_{t}(u, v)$ of $\left(y_{t+1}, z_{t+1}\right)$, given $I_{t}$, is given by :

$$
\begin{aligned}
\varphi_{t}(u, v) & =E\left[\exp \left(u y_{t+1}+v^{\prime} z_{t+1}\right) \mid \underline{y_{t}}, \underline{z_{t}}\right] \\
& =E\left[E\left[\exp \left(u y_{t+1}+v^{\prime} z_{t+1}\right) \mid z_{t+1}, \underline{y_{t}}, \underline{z_{t}}\right] \mid \underline{y_{t}}, \underline{z_{t}}\right] \\
& =E\left[\exp \left(v^{\prime} z_{t+1}\right) E\left[\exp \left(u y_{t+1}\right) \mid \underline{y_{t}}, \underline{z_{t+1}}\right] \mid \underline{y_{t}}, \underline{z_{t}}\right] \\
& =E\left[\exp \left(v^{\prime} z_{t+1}+u \mu\left(\underline{y_{t}}, \underline{z_{t+1}}\right)+\frac{1}{2} u^{2} \sigma^{2}\left(\underline{y_{t}}, \underline{z_{t+1}}\right)\right) \mid \underline{y_{t}}, \underline{z_{t}}\right] \\
& =\sum_{z_{t+1}} p_{t}\left(z_{t+1}\right) \exp \left(v^{\prime} z_{t+1}+u \mu\left(\underline{y_{t}}, \underline{z_{t+1}}\right)+\frac{1}{2} u^{2} \sigma^{2}\left(\underline{y_{t}}, \underline{z_{t+1}}\right)\right),
\end{aligned}
$$

with $p_{t}\left(z_{t+1}\right)=f\left(z_{t+1} \mid \underline{y_{t}}, \underline{z_{t}}\right)$.

Proof of Proposition 17 : The conditional risk-neutral distribution $Q_{t}$ of $\left(y_{t+1}, z_{t+1}\right)$, given $I_{t}$, has a Laplace transform given by :

$$
\begin{aligned}
& \varphi^{Q_{t}}(u, v) \\
& =\frac{\varphi_{t}\left(\alpha_{t}+u, \delta_{t}+v\right)}{\varphi_{t}\left(\alpha_{t}, \delta_{t}\right)} \\
& =\frac{\sum_{z_{t+1}} p_{t}\left(z_{t+1}\right) \exp \left(\left(\delta_{t}+v\right)^{\prime} z_{t+1}+\left(\alpha_{t}+u\right) \mu\left(\underline{y_{t}}, \underline{z_{t+1}}\right)+\frac{1}{2}\left(\alpha_{t}+u\right)^{2} \sigma^{2}\left(\underline{y_{t}}, \underline{z_{t+1}}\right)\right)}{\sum_{z_{t+1}} p_{t}\left(z_{t+1}\right) \exp \left(\delta_{t}^{\prime} z_{t+1}+\alpha_{t} \mu\left(\underline{y_{t}}, \underline{z_{t+1}}\right)+\frac{1}{2} \alpha_{t}^{2} \sigma^{2}\left(\underline{y_{t}}, \underline{z_{t+1}}\right)\right)} \\
& =\sum_{z_{t+1}} \nu_{t}\left(z_{t+1}\right) \exp \left[v^{\prime} z_{t+1}+u\left[\mu\left(\underline{y_{t}}, \underline{z_{t+1}}\right)+\alpha_{t} \sigma^{2}\left(\underline{y_{t}}, \underline{z_{t+1}}\right)\right]+\frac{1}{2} u^{2} \sigma^{2}\left(\underline{y_{t}}, \underline{z_{t+1}}\right)\right],
\end{aligned}
$$

where

$$
\nu_{t}\left(z_{t+1}\right)=\frac{p_{t}\left(z_{t+1}\right) \exp \left(\delta_{t}^{\prime} z_{t+1}+\alpha_{t} \mu\left(\underline{y_{t}}, \underline{z_{t+1}}\right)+\frac{1}{2} \alpha_{t}^{2} \sigma^{2}\left(\underline{y_{t}}, \underline{z_{t+1}}\right)\right)}{\sum_{z_{t+1}} p_{t}\left(z_{t+1}\right) \exp \left(\delta_{t}^{\prime} z_{t+1}+\alpha_{t} \mu\left(\underline{y_{t}}, \underline{z_{t+1}}\right)+\frac{1}{2} \alpha_{t}^{2} \sigma^{2}\left(\underline{y_{t}}, \underline{z_{t+1}}\right)\right)} .
$$




\section{R E F E R E N C E S}

Abel, A. (1994) : "Exact Solution of Expected Rates of Return Under Markov RegimeSwitching : Implication for the Equity Premium", Journal of Money, Credit and Banking, 26, 345-361.

Abel, A. (1999) : "Risk Premia and Term Premia in General Equilibrium", Journal of Monetary Economics, 43, 3-33.

Adler, R. J., Feldman, R., and M. Taqqu (1998) : "A Practical Guide to Heavy Tails: Statistical Techniques and Applications", Birkhäuser, Boston, Basel, Berlin.

Ait-Sahalia, Y., and J. Duarte (2003) : "Nonparametric Option Pricing Under Shape Restrictions", Journal of Econometrics, 116, 9-47.

Ait-Sahalia, Y., and A. Lo (1998) : "Nonparametric Estimation of State-Price Densities Implicit in Financial Asset Prices", The Journal of Finance, 53, 499-547.

Akgiray, V., and G. G. Booth (1987) : "Compound Distribution Models of Stock Returns: An Empirical Comparison", The Journal of Financial Research, 10, 269-280.

Amin, K. I., and V. K. Ng (1993) : "Option Valuation with Systematic Stochastic Volatility", The Journal of Finance, 48, 881-910.

Andersen, T., Benzoni, L., and J. Lund (2002) : "An Empirical Investigation of ContinuousTime Equity Return Models", The Journal of Finance, 57, 1239-1284.

Barndorff-Nielsen, O. E. (1994) : "Gaussian Inverse Gaussian Processes and the Modeling of Stock Returns", Technical Report, Aarhus University.

Bauwens, L., Bos, C. S., and H. K. van Dijk (1999) : "Adaptive Polar Sampling with an Application to Bayes Measure of Value-at-Risk", Tinberg Institute Discussion Paper TI 99-082/4, Erasmus University Rotterdam.

Bollerslev, T. (1987) : "A Conditional Heteroskedastic Time Series Model for Speculative Prices and Rates of Return", Review of Economics and Statistics, 69, 542-547.

Baillie, R. T., and T. Bollerslev (1989) : "The Message in Daily Exchange Rates: A ConditionalVariance Tale", The Journal of Business and Economic Statistics, 7, 297-305.

Bakshi, G., Cao, C., and Z. Chen (1997) : "Empirical Performance of Alternative Option Pricing Models", The Journal of Finance, 52, 2003-2049.

Bakshi, G., and D. Madan (2000) : "Spanning and Derivative-Security Valuation", Journal of Financial Economics, 55, 205-238.

Bakshi, G., Kapadia, N., and D. Madan (2003) : "Stock Return Characteristics, Skew Laws, and the Differential Pricing of Individual Equity Options", The Review of Financial Studies, 16, 101-143. 
Bates, D. S. (1996a) : "Jumps and Stochastic Volatility: Exchange Rate Processes Implicit in Deutsche Mark Options", The Review of Financial Studies, 9, 69-107.

Bates, D. S. (1996b) : "Dollar jump fears, 1984-1992 : distributional abnormalities implicit in currency futures options", Journal of International Money and Finance, 15, 65-93.

Bates, D. S. (2000) : "Post-'87 crash fears in the S\&P 500 Futures Option Market", Journal of Econometrics, 94, 181-238.

Billio, M., and L. Pelizzon (2000) : "Option Smile and Switching Volatility", GRETA (Venice, Italy), Working Paper n. 00.01.

Black, F., and M. Scholes (1973) : "The Pricing of Options and Corporate Liabilities", Journal of Political Economy, 81, 637-659.

Bollen, N. P. B. (1998) : "Valuing Options in Regime-Switching Models", Journal of Derivatives, 6, 38-49.

Bollerslev, T., and H. O. Mikkelsen (1999) : "Long-Term Equity Anticipation Securities and Stock Market Volatility Dynamics", Journal of Econometrics, 92, 75-99.

Bonomo, M., and R. Garcia (1994) : "Can a Well-Fitted Equilibrium Asset Pricing Model Produce Mean Reversion?", Journal of Applied Econometrics, 9, 19-29.

Bonomo, M., and R. Garcia (1996) : "Consumption and Equilibrium Asset Pricing : An Empirical Assessment", Journal of Empirical Finance, 3, 239-265.

Brännäs, K., and N. Nordman (2001) : "Conditional Skewness Modeling for Stock Returns", Umea Economic Studies, 562, Department of Economics, Umea University, Sweden.

Broadie, M., Chernov, M., and M. Johannes (2004) : "Model Specification and Risk Premiums : The Evidence from Futures Options".

Calvet, L., and A., Fisher (2004) : "Asset Prices with Multifrequency Regime-Switching and Learning : A Volatility Specification", Department of Economics, Harvard University.

Camara, A. (2003) : "A Generalization of the Brennan-Rubinstein Approach for the Pricing of Derivatives", The Journal of Finance, 58, 805-819.

Campbell, J., Lo, A. W., and A. C. MacKinlay (1997) : "The Econometrics of Financial Markets", Princeton University Press.

Campbell, S. D., and C. Li (2002) : "Option Pricing in Regime-Switching Environments", Working Paper.

Carr, P., Chang, E. C., and D. Madan (1998) : "The Variance Gamma Process and Option Pricing", R. H. Smith School of Business, University of Maryland.

Carr, P., L. Wu (2003) : "The Finite Moment Log Stable Process and Option Pricing", The Journal of Finance, 58, 753-777. 
Carr, P., L. Wu (2004) : "Time-Changed Lévy Processes and Option Pricing", Journal of Financial Economics, 71, 113-141.

Cecchetti, S., Lam, P.-S., and N. Mark (1990) : "Mean Reversion in Equilibrium Asset Prices", American Economic Review, 80, 398-418.

Chesney, M., and L. Scott (1989) : "Pricing European Currency Options: A Comparison of the Modified Black-Scholes Model and a Random Variance Model", The Journal of Financial and Quantitative Analysis, 24, 267-284.

Chernov, M., and E. Ghysels (2000) : "A Study Towards a Unified Approach to the Joint Estimation of Objective and Risk Neutral Measures for the Purpose of Option Pricing", Journal of Financial Economics, 56, 407-458.

Chernov, M., Gallant, R., Ghysels, E., and G. Tauchen (2003) : "Alternative Models for Stock Price Dynamics", Journal of Econometrics, 116, 225-257.

Chourdakis, K., and E. Tzavalis (2000) : "Option Pricing under Discrete Shifts in Stock Returns", Queen Mary University of London, Department of Economics, Working Paper No. 426.

Christoffersen, P., Heston, S., and K. Jacobs (2004) : "Option Valuation with Conditional Skewness", Faculty of Management, McGill University and CIRANO.

Christoffersen, P., and K. Jacobs (2004) : "Which GARCH Model for Option Valuation?", Management Science, 50, 1204-1221.

Christoffersen, P., Jacobs, K., and Y. Wang (2005) : "Option Valuation with Long-run and Short-run Volatility Components", Faculty of Management, McGill University and CIRANO.

Christoffersen, P., Elkamhi, R., and K. Jacobs (2005) :"No-Arbitrage Valuation of Contingent Claims in Discrete Time", Faculty of Management, McGill University and CIRANO.

Cochrane, J. H. (2001) : "Asset Pricing", Princeton University Press.

Cont, R. and J. da Fonseca (2002) : "Dynamics of Implied Volatility Surfaces", Quantitative Finance, 2, 45-60.

Duan, J.-C. (1995) : "The GARCH Option Pricing Model", Mathematical Finance, 5, 13-32.

Duan, J.-C. (1996) : "A Unified Theory of Option Pricing Under Stochastic Volatility - From GARCH to Diffusion", Hong Kong University of Science and Technology, Working Paper.

Duan, J.-C. (1999) : "Conditionally Fat-Tailed Distributions and the Volatility Smile in Options", Rotman School of Management, University of Toronto.

Duan, J.-C. (2002) : "Nonparametric Option Pricing by Transformation", Rotman School of Management, University of Toronto.

Duan, J.-C., Gauthier, G., Simonato, J. G., and C. Sasseville (2004): "Approximate the GJRGARCH and EGARCH Option Pricing Models Analytically", Rotman School of Management, 
University of Toronto.

Duan, J.-C., Popova, I., and P. Ritchken (2002) : "Option Pricing under Regime Switching", Quantitative Finance, 2, 116-132.

Duan, J.-C., Ritchken, P., and Z. Sun (2005a) : "Jump Starting GARCH: Pricing and Hedging Options with Jumps in Returns and Volatilities", Rotman School of Management, University of Toronto.

Duan, J.-C., Ritchken, P., and Z. Sun (2005b) : "Approximating GARCH-Jump Models, JumpDiffusion Processes, and Option Pricing" (to appear in Mathematical Finance), Rotman School of Management, University of Toronto.

Duffie, D., J. Pan, and K. Singleton (2000) : "Transform Analysis and Asset Pricing for Affine Jump Diffusions", Econometrica, 68, 1343-1376.

Dumas, B., Fleming, J., and R. E. Whaley (1998) : "Implied Volatility Functions: Empirical Tests", The Journal of Finance, 6, 2059-2106.

Eberlein, E., and K. Keller (1995) : "Hyperbolic Distributions in Finance", Bernoulli, 1, 281299.

Engle, R., and C. Mustafa (1992) : "Implied ARCH Models from Option Prices", Journal of Econometrics, 52, 289-311.

Epstein, L., and S. Zin (1989) : "Substitution, Risk Aversion and the Temporal Behavior of Consumption and Asset Returns : A Theoretical Framework", Econometrica, 57, 937-968.

Eraker, B., Johannes, M., and N. Polson (2003) : "The Impact of Jumps in Volatility and Returns", The Journal of Finance, 58, 1269-1300.

Eraker, B. (2004) : "Do Stock Prices and Volatility Jump? Reconciling Evidence from Spot and Option Prices", The Journal of Finance, 59, 1367-1403.

Fama, E. (1965) : "The Behavior of Stock Market Prices", Journal of Business, 38, 34-105.

Gallant, A. R., and D. W. Nychka (1987) : "Seminonparametric maximum likelihood estimation", Econometrica, 55, 363-390.

Garcia, R., and E. Renault (1998) : "Risk Aversion, Intertemporal Substitution, and Option Pricing", Working Paper Cirano.

Garcia, R., Ghysels, E., and E. Renault (2002) : "The Econometrics of Option Pricing", Working Paper Cirano.

Garcia, R., Luger, R., and E. Renault (2001) : "Asymmetric Smiles, Leverage Effects and Structural Parameters", Working Paper Cirano.

Garcia, R., Luger, R., and E. Renault (2003) : "Empirical Assessment of an Intertemporal Option Pricing Model with Latent Variables", Journal of Econometrics, 116, 49-83. 
Garcia, R., Meddahi, N., and R. Tedongap (2006) : "An Analytical Framework for Assessing Asset Pricing Models and Predictability", Working Paper, Université de Montréal.

Gerber, H. U., and E. S. W. Shiu (1994) : "Option Pricing by Esscher Transforms", Transactions of Society of Actuaries, 46, 99-191.

Ghysels, E., Patilea, V., Renault, E., and O. Torrès (1997) : "Nonparametric Methods and Option Pricing", Working Paper Cirano.

Gordon, S., and P. St-Amour (2000) : "A Preference Regime Model of Bear and Bull Markets", American Economic Review, 90, 1019-1033.

Gourieroux, C., and A. Monfort (2006) : "Econometric Specifications of Stochastic Discount Factor Models", forthcoming Journal of Econometrics.

Gourieroux, C., Monfort, A., and V. Polimenis (2002) : "Affine Term Structure Models", CREST DP.

Gourieroux, C., Monfort, A., and V. Polimenis (2006) : "Affine Models for Credit Risk Analysis", forthcoming Journal of Financial Econometrics.

Hamilton, J. D. (1989) : "A New Approach to the Economic Analysis of Nonstationary Time Series and the Business Cycle", Econometrica, 57, 357-384.

Hansen, B. E. (1994) : "Autoregressive Conditional Density Estimation", International Economic Review, 35, 705-730.

Hansen, L. P., and S. Richard (1987) : "The Role of Conditioning Information in Deducing Testable Restrictions Implied by Dynamic Asset Pricing Models", Econometrica, 55, 587-613.

Harvey, C. R., and A. Siddique (1999) : "Autoregressive Conditional Skewness", Journal of Financial and Quantitative Analysis, 34, 465-487.

Hass, M., Mittnik, S., and M. Paolella (2002) : "Mixed Normal Conditional Heteroskedasticity", Working Paper, Institute of Statistics and Econometrics, University of Kiel, Germany.

Heston, S. L. (1993) : "A Closed-Form Solution for Options with Stochastic Volatility with Applications to Bond and Currency Options", The Review of Financial Studies, 6, 327-343.

Heston, S. L., and S. Nandi (2000) : "A Closed-Form GARCH Option Valuation Model", Review of Financial Studies, 13, 585-625.

Huang, J.-Z., L. Wu (2004) : "Specification Analysis of Option Pricing Models Based on TimeChanged Lévy Processes", The Journal of Finance, 59, 1405-1439.

Hull, J., and A. White (1987) : "The Pricing of Options on Assets with Stochastic Volatilities", Journal of Finance, 42, 281-300.

Jondeau, E., and M. Rockinger (2000) : "Reading the Smile: The Message Conveyed by the Methods which Infer Risk Neutral Densities", Journal of International Money and Finance, 19, 
885-915.

Jondeau, E., and M. Rockinger (2001) : "Gram-Charlier densities", Journal of Economic Dynamics and Control, 25, 1457-1483.

Jondeau, E., and M. Rockinger (2002) : "Entropy densities with an application to autoregressive conditional skewness and kurtosis", Journal of Econometrics, 106, 119-142.

Jondeau, E., and M. Rockinger (2003) : "Conditional volatility, skewness, and kurtosis : existence, persistence, and comovements", Journal of Economic Dynamics and Control, 27, 1699-1737.

Jones, C. (2003) : "The Dynamics of Stochastic Volatility : Evidence from Underlying and Option Markets", Journal of Econometrics, 116, 181-224.

Kon, S. J. (1984) : "Models of Stock Returns: A Comparison", The Journal of Finance, 39, 147-165.

Kuechler, U., Neumann, K., Soerensen, M., and A. Streller (1999) : "Stock Returns and Hyperbolic Distributions", Mathematical and Computer Modeling, 29, 1-15.

Lambert, P., and S. Laurent (2000) : "Modeling Skewness Dynamics in Series of Financial Data using Skewed Location-Scale Distributions", Working Paper.

Lambert, P., and S. Laurent (2001) : "Central Bank Interventions and Jumps in Double Long Memory Models of Daily Exchange Rates", Working Paper.

Léon, A., Mencia, J., and E. Sentana (2005) : "Parametric Properties of Semi-nonparametric Distributions, with Application to Option Valuation", Working Paper CEMFI.

Lin, B. H., and S. K. Yeh (2000) : "On the Distribution and Conditional Heteroskedasticity in Taiwan Stock Prices", Journal of Multinational Financial Management, 10, 367-395.

Madan, D., and F. Milne (1994) : "Contingent Claims Valued and Hedged by Pricing and Investing in a Basis", Mathematical Finance, 4, 223-245.

Madan, D., and E. Seneta (1990) : "The Variance Gamma (V. G.) Model for Share Market Returns", Journal of Business, 63, 511-524.

Mandelbrot, B. (1962) : "Paretian Distributions and Income Maximization", Quarterly Journal of Economics, 76, 57-85.

Mandelbrot, B. (1963a) : "New Methods in Statistical Economics", Journal of Political Economy, 71, 421-440.

Mandelbrot, B. (1963b) : "The Variation of Certain Speculative Prices", Journal of Business, $36,394-419$.

Mandelbrot, B. (1967) : "The Variation of some Other Speculative Prices", Journal of Business, 40, 393-413. 
Mandelbrot, B. (1997) : "Fractal and Scaling in Finance. Discontinuity, Concentration, Risk", Springer, New York, Berlin, Heidelberg.

McLachlan, G., and D. Peel (2000) : "Finite Mixture Models", John Wiley \& Sons, New York.

Melick, W. R., and C. P. Thomas (1997) : "Recovering an Asset's Implied PDF from Option Prices: An Application to Crude Oil during the Gulf Crisis", The Journal of Financial and Quantitative Analysis, 32, 91-115.

Melino, A., and S. Turnbull (1990) : "Pricing Foreign Currency Options with Stochastic Volatility", Journal of Econometrics, 45, 239-265.

Melino, A., and A. X. Yang (2003) : "State Dependent Preferences Can Explain the Equity Premium Puzzle", Department of Economics, University of Toronto.

Merton, R. C. (1976) : "Option Pricing When the Underlying Stock Returns are Discontinuous", Journal of Financial Economics, 3, 125-144.

Mittnick, S., and S. T. Rachev (1993a) : "Modeling Asset Returns with Alternative Stable Distributions", Econometric Review, 12, 261-330.

Mittnick, S., and S. T. Rachev (1993b) : "Reply to Comments on Modeling Asset Returns with Alternative Stable Distributions and some extensions", Econometric Review, 12, 347-389.

Mittnick, S., Paolella, M. S., and S. T. Rachev (1993a) : "Modeling the Persistence of Conditional Volatilities with GARCH-stable Processes", Technical Report, University of California, Santa Barbara.

Naik, V. (1993) : "Option Valuation and Hedging Strategies with Jumps in the Volatility of Asset Returns", The Journal of Finance, 48, 1969-1984.

Palm, F. C., and P. J. G. Vlaar (1993) : "The Message in Weekly Exchange Rates in the European Monetary System: Mean Reversion, Conditional Heteroskedasticity, and Jumps", Journal of Business and Economic Statistics, 11, 351-360.

Palm, F. C., and P. J. G. Vlaar (1997) : "Simple Diagnostic Procedures for Modeling Financial Time Series", Allgemeines Statistiches Archiv 81, 85-101.

Pan, J. (2002) : "The Jump-Risk Premia Implicit in Options : Evidence from an Integrated Time-Series Study", Journal of Financial Economics, 63, 3-50.

Paolella, M. (1999) : "Tail Estimation and Conditional Modeling of Heterosckedastic Time Series", Ph.D. Thesis, Institute of Statistics and Econometrics, University of Kiel.

Renault, E., and N. Touzi (1996) : "Option Hedging and Implied Volatilities in a Stochastic Volatility Model", Mathematical Finance, 6, 279-302.

Ritchey, R. (1990) : "Call Option Valuation for Discrete Normal Mixtures", Journal of Financial Research, 13, 285-296. 
Rockinger, M., and E. Jondeau (2002) : "Entropy Densities with Application to Autoregressive Conditional Skewness and Kurtosis", Journal of Econometrics, 106, 119-142.

Rubinstein, M. (1994) : "Implied Binomial Trees", The Journal of Finance, 49, 771-818.

Scott, L. (1987) : "Option Pricing when the Variance Changes Randomly : Theory, Estimation, and an Application", The Journal of Financial and Quantitative Analysis, 22, 419-438.

Stein, E. M., and J. C. Stein (1991) : "Stock Price Distributions with Stochastic Volatility: An Analytic Approach", The Review of Financial Studies, 4, 727-752.

Stutzer, M. (1996) : "A Simple Nonparametric Approach to Derivative Security Valuation", The Journal of Finance 51(5), 1633-1652.

Tucker, A. L. (1992) : "A Reexamination of Finite- and Infinite-Variance Distributions as Models of Daily Stock Returns", Journal of Business \& Economic Statistics, 10, 1, 73-81.

Tucker, A. L., and L. Pond (1998) : "The Probability Distribution of Foreign Exchange Price Changes: Tests of Candidate Processes", Review of Economics and Statistics, 11, 638-647.

Veronesi, P. (1999) : "Stock Market Overreaction to Bad News in Good Times : A Rational Expectations Equilibrium Model", The Review of Financial Studies, 12, 975-1007.

Veronesi, P. (2000) : "How Does Information Quality Affect Stock Returns?", The Journal of Finance, 55, 807-837.

Veronesi, P. (2004) : "The Peso Problem Hypothesis and Stock Market Returns", Journal of Economic Dynamics and Control, 28, 707-725.

Whitelaw, R. F. (2000) : "Stock Market Risk and Return : An Equilibrium Approach", The Review of Financial Studies, 13, 521-547. 
FIGURE 1 - Implied Volatility

mu1 $=$ mu2 $=.03$, var $=.04, p=0.50$, var2 $=$ from .04 to .07

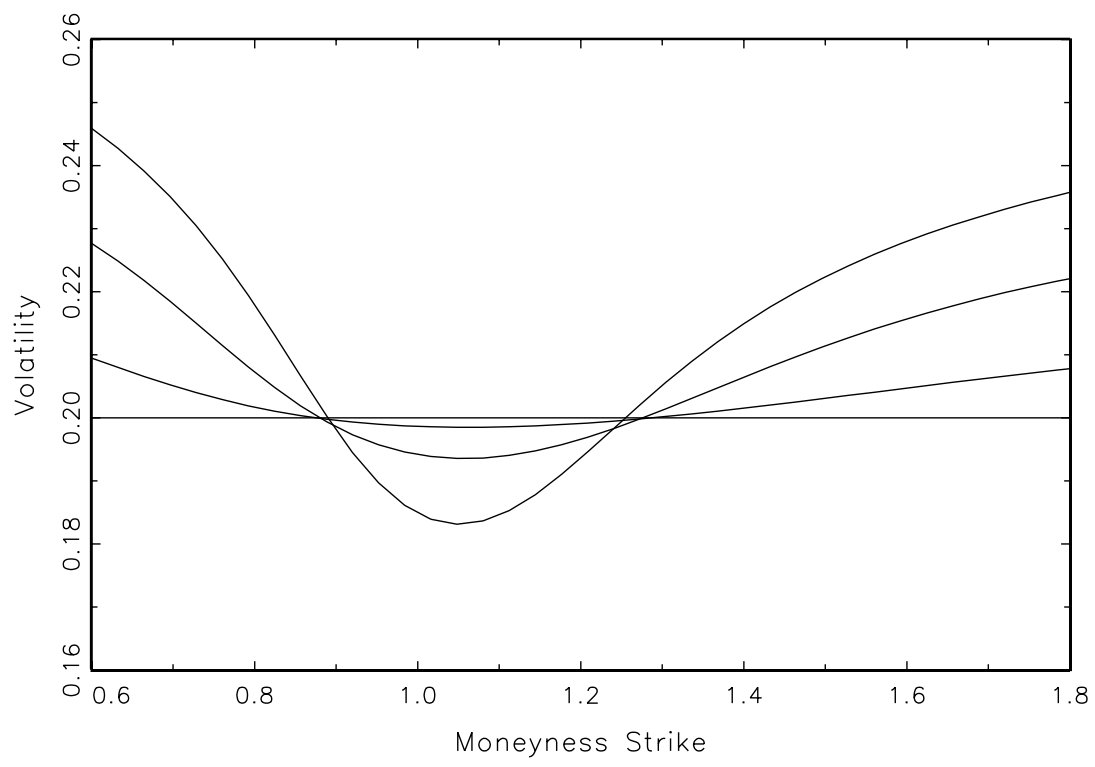

FIGURE 2 - Implied Volatility

mu1 $=$ mu2 $=.07$, var $=.05, p=0.90$, var2 $=$ from .05 to .11

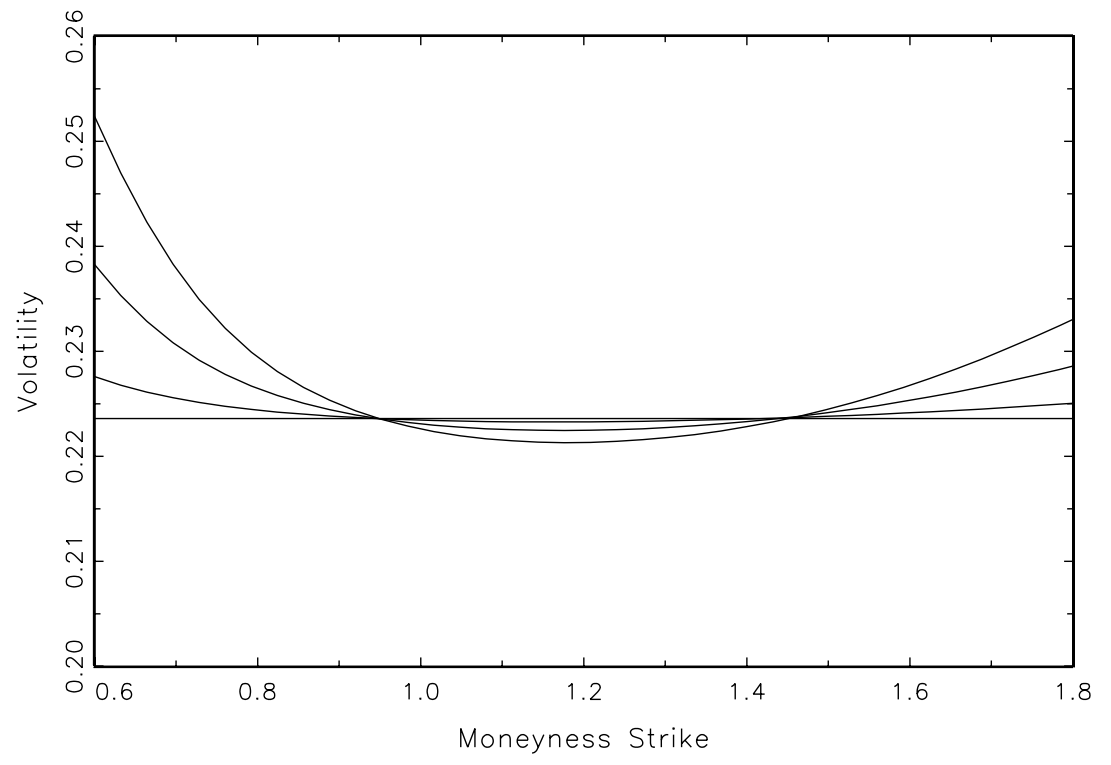


FIGURE 3 - Implied Volatility

mu1 $=.01$, mu2 $=.06$, var $=.06, p=0.5$, var $1=$ from .07 to .09

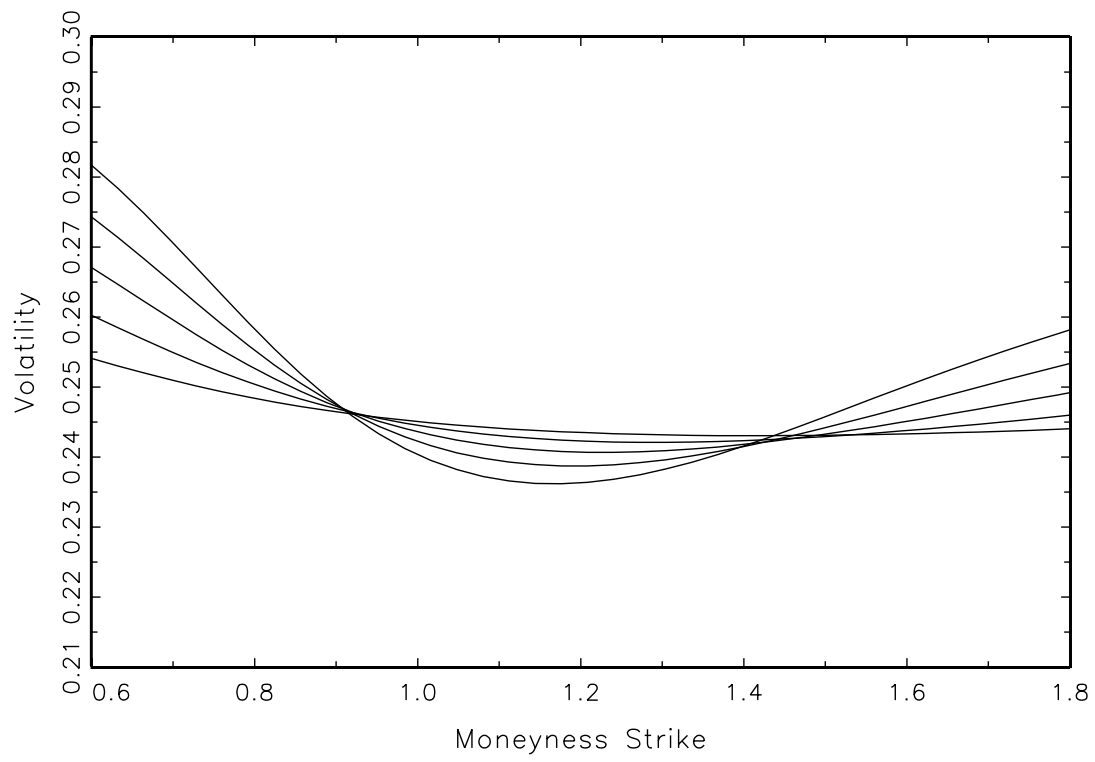


FIGURE 4 - Implied Volatility Surface

mu1 $=$ mu2 $=0.03, \operatorname{var}=.025, \operatorname{var} 2=.035, p=0.5$

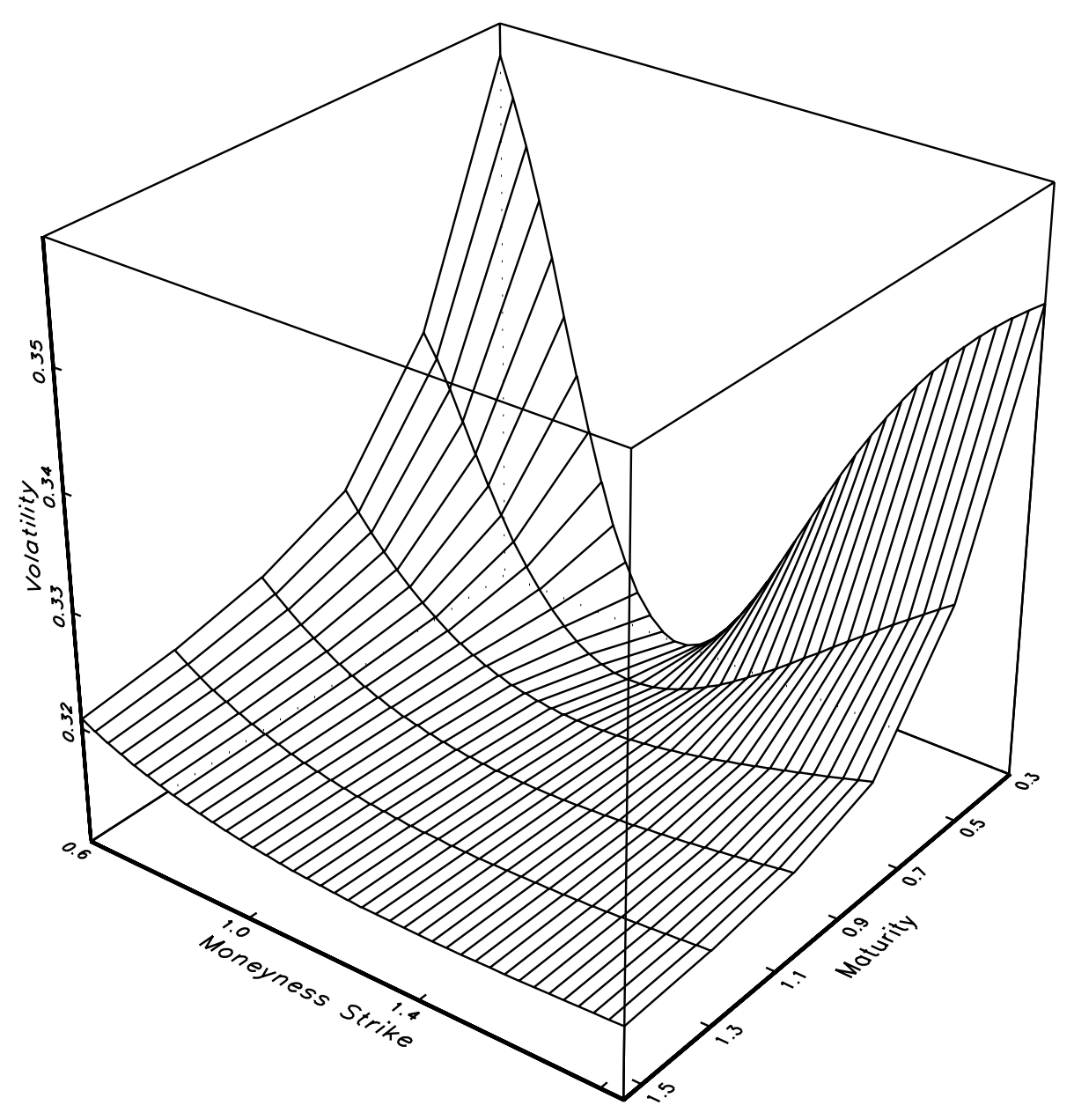


FIGURE 5 - Implied Volatility

mu1 $=.03$, mu2 $=.07, \operatorname{var} 2=.04, p=0.5, \operatorname{var} 1=.040$ (bottom) to .080 (up)

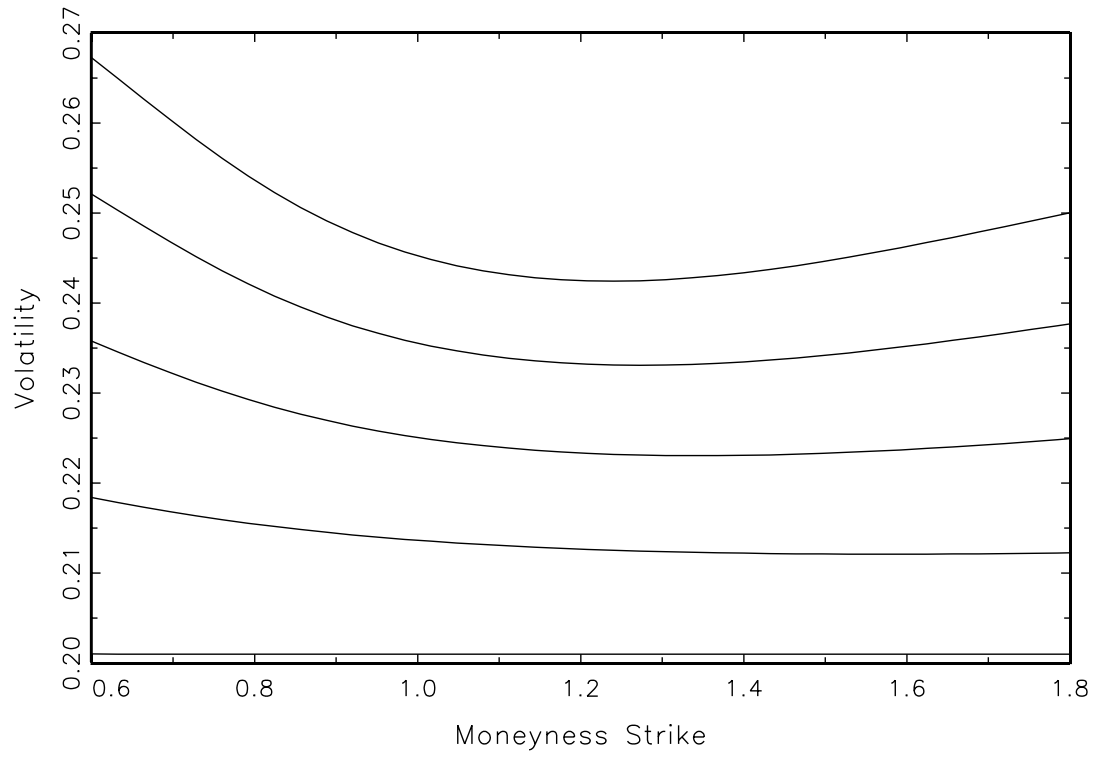

FIGURE 6 - Implied Volatility for Extreme Risks

mu $1=$ mu $2=.07$, var $=.06$

lambda $=.5001$ (flat curve) to .001 (up)

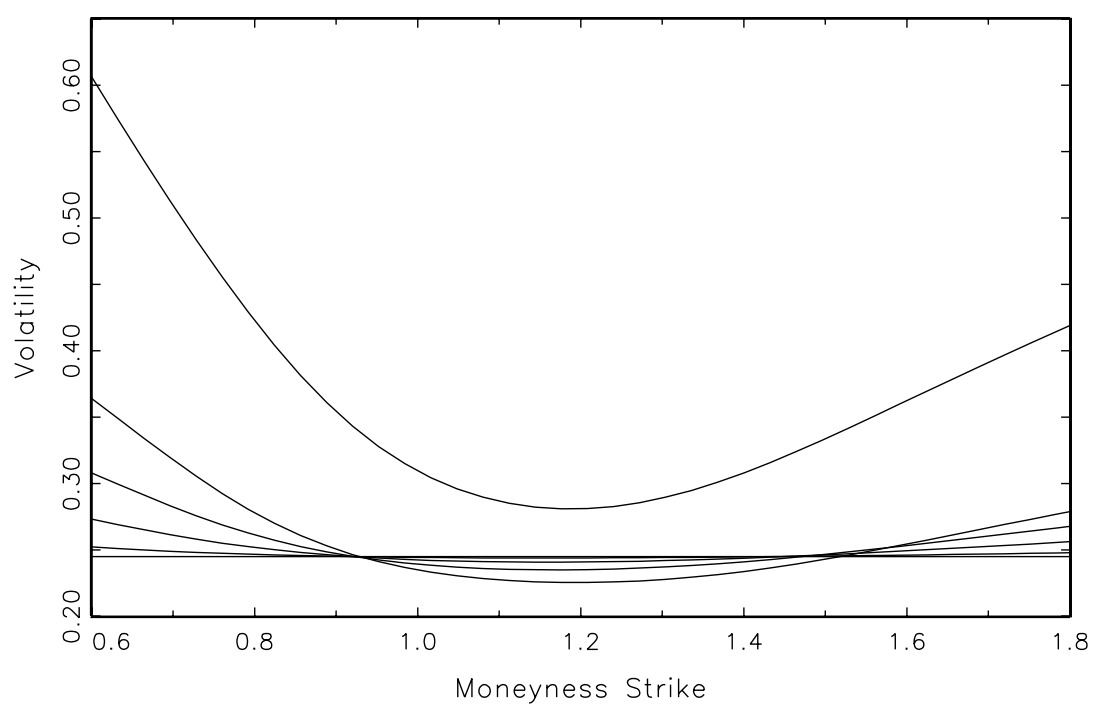


FIGURE 7 - Implied Volatility for Extreme Risks:

relation with lambda (strong kurtosis effect)

$\mathrm{mu} 1=\mathrm{mu} 2=.07, \operatorname{var}=.06, \mathrm{k}=1$

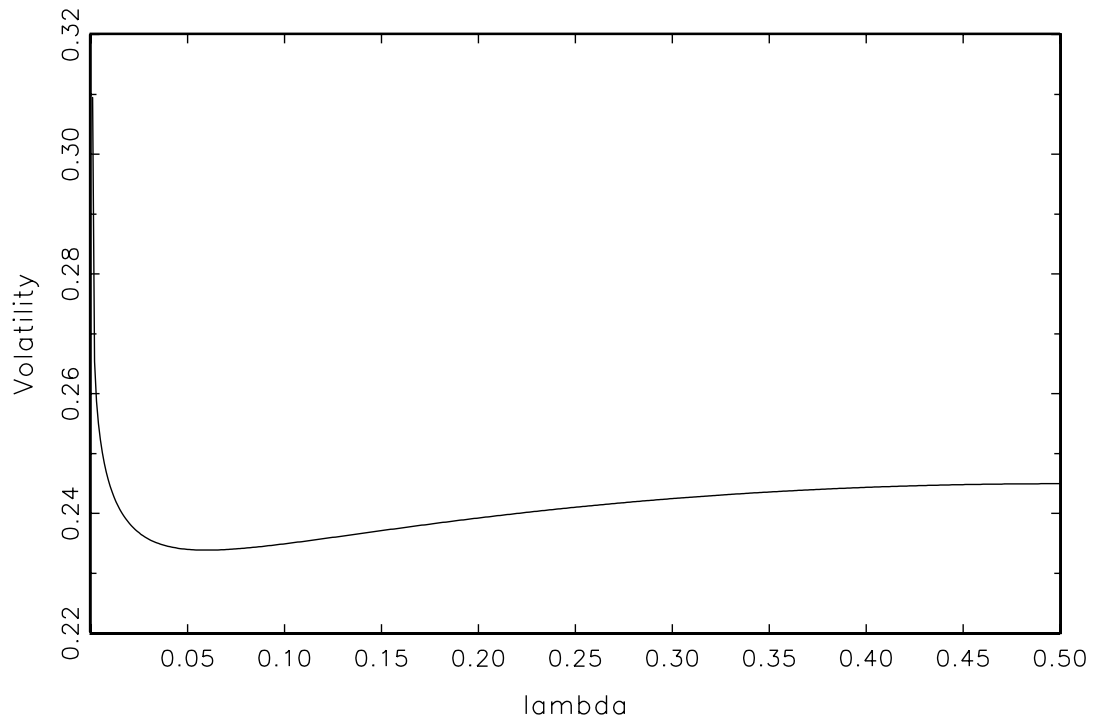


FIGURE 8 - Regime Switching Option Pricing Model Implied Volatility Surface

$m u 1=m u 2=.03, \operatorname{var} 1=.03, \operatorname{var} 2=.04, \operatorname{pi} 11=0.99, \operatorname{pi} 22=0.95$

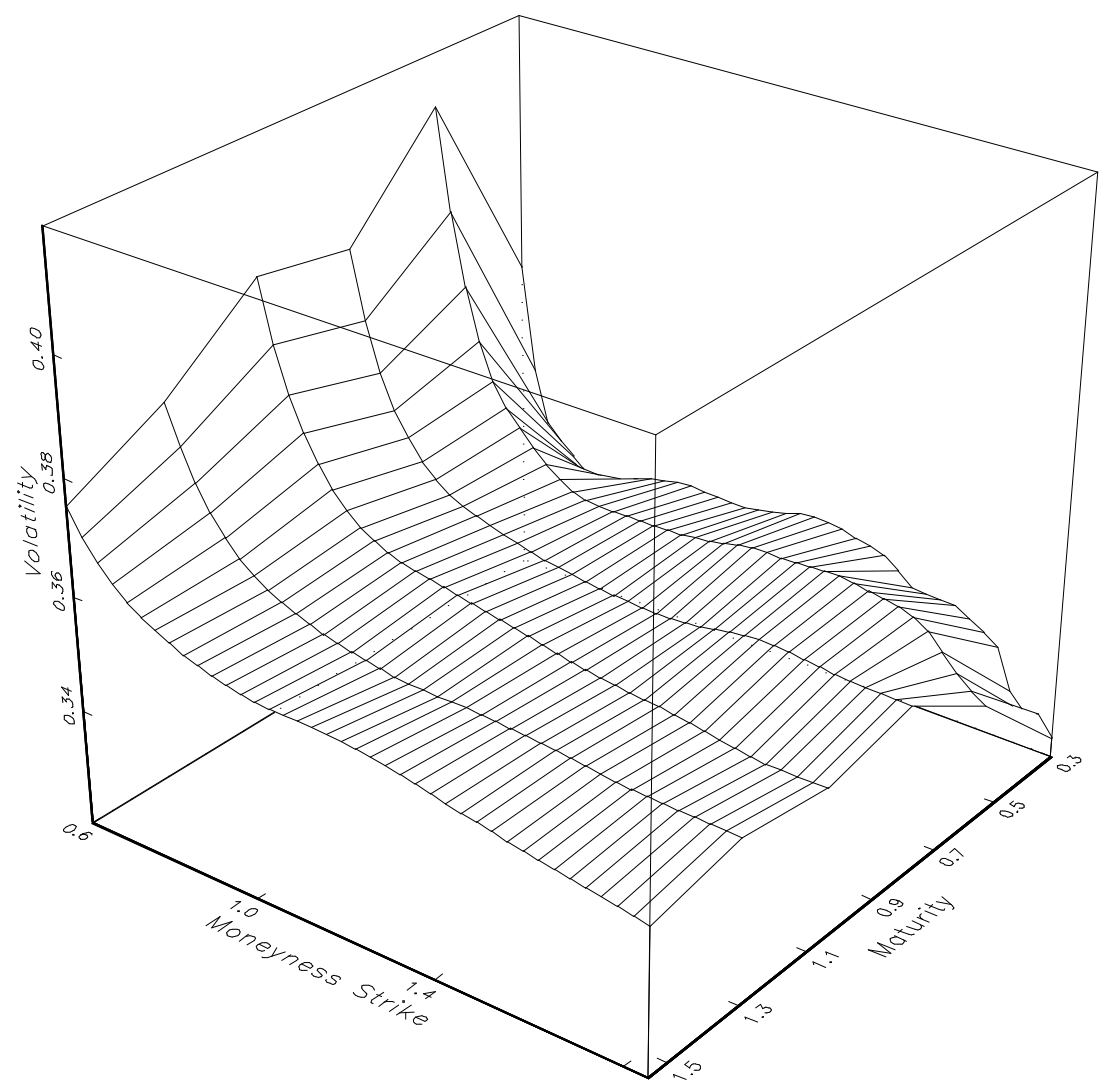

